\title{
Viscoelastic Computations of Polymeric Wire-coating Flows
}

\author{
H. Matallah, P. Townsend and M.F. Webster \\ Institute of Non-Newtonian Fluid Mechanics \\ University of Wales, Swansea SA2 8PP
}

March 6, 2002

\begin{abstract}
This study considers both a single and multi-mode viscoelastic analysis for wirecoating flows. The numerical simulations utilise a finite element time-stepping technique, a Taylor-Petrov-Galerkin/pressure-correction scheme employing both coupled and decoupled procedures between stress and kinematic fields. An exponential Phan-Thien/Tanner model is used to predict pressure-drop and residual stress for this process. Rheometrical data fitting is performed for steady shear and pure extensional flows, considering both high and low density polyethylene melts. Simulations are conducted to match experimental pressure-drop/flowrate data for a contraction flow. Then, for a complex industrial wire-coating flow, stress and pressure drop are predicted numerically and quantified. The benefits are extolled of the use of a multi-mode model that can incorporate a wide-range discrete relaxation spectrum to represent flow response in complex settings. Contrast is made between LDPE and HDPE polymers, and dependency on individual relaxation modes is identified in its contribution to overall flow behaviour.
\end{abstract}

Keywords: Wire-coating, tube-tooling, multi-mode, finite element, Taylor-Galerkin, Recovery

\section{INTRODUCTION}

In the field of polymer coating of wires, experimental studies are extremely difficult to perform due to the small size of the dies and minute volume of polymer melt within the region of interest. This leads to the need for numerical simulation as a predictive aid to optimise the process via die design and flow modelling. This allows for the analysis of variation in certain key parameters, without having to resort to trial-and error expensive and difficult experiments. There are two basic types of cable or wire-coating die designs commonly employed. These are represented schematically in Figure 1, and are termed pressure and tube-tooling designs. In pressure-tooling, the melt is driven under pressure making contact with the wire inside the die. In contrast, for tube-tooling, the melt is drawn down by the motion of the wire and the melt is extruded beyond the die. In both instances, the geometry is annular in cross section. The setting of the flow geometry is found to be crucial in obtaining optimal coatings.

There have been a number of studies that have addressed the modelling of wire-coating flows; see for example Fenner and Williams [1], Caswell and Tanner [2], Mitsoulis et al. [3, 4], Huang et al. [5], Binding et al. [6] for pressure-tooling, and Gunter et al. [7], Mutlu et al. $[8,9,10]$ for tube-tooling. These have provided some progress within the inelastic, nonisothermal and viscoelastic regimes. In a wider flow and processing context, some recent 
attempts have been made to embrace differential multi-mode simulations, see for example the works of Baaijens [11], Baaijens et al. [12], Azaiez et al. [13] and Gupta et al. [14]. Such a multi-mode approach for differential constitutive models is more costly, yet can provide a more accurate representation of the material rheology. The present study addresses the significance of employing a multi-mode, as opposed to a single-mode, differential constitutive modelling approach to predict numerically the behaviour of tube-tooling wire-coating flows for two polymer melts, low and high density polyethylene. We concentrate on the quality and significance of numerical predictions attainable from multi-mode approximations in contrast to single-mode alternatives. The modelling assumes incompressible melt flow, isothermal conditions, no-slip within the die, and an estimated location for the free-surfaces, see Refs. $[8,9]$. Practical experience from the process itself provides this location.

We are concerned with two parts of the tube-tooling wire-coating process, namely flow within the die itself and a draw-down flow beyond the die onto the wire cable. The flow is generated by a pressure head and the dragging action of the moving wire. This type of tooling design is prevalent for wide bore cables, or outer coatings of multi-cable combinations. Cable speed is taken as $0.334 \mathrm{~m} / \mathrm{s}$. The main goal of modelling such a complex industrial problem is to achieve process optimisation. This is governed typically by minimisation of the flow-induced residual stress locked into the coating, and in addition, acceptable levels of pressure-drop across the die. The flow response of the coating material and the die design [15] both have a role to play in this optimisation procedure. Pressure-drop predictions provide some means of correspondence between simulation and practice.

An exponential Phan-Thien/Tanner (PTT) differential constitutive model is selected to represent the rheometrical behaviour of low and high density polyethylene melts (LDPE and HDPE) in steady shear and uniaxial flow. Typically, such material samples display both shear thinning and strain softening properties and an exponential PTT model is capable of reproducing such behaviour in qualitative form $[16,17]$. Simple shear and uniaxial flows are used to evaluate the PTT parameter set $(\epsilon, \xi)$, for which a close fit to the experimental data must be established. To calibrate pressure drops as a function of flowrate and compare experimental with predicted observations, a nineteen to one axi-symmetric contraction flow is adopted. For this flow, both single and multi-mode models are implemented. Once optimality in parameter set is established, we turn attention to tube-tooling flow and the multi-mode simulations for LDPE and HDPE polymer coatings.

The numerical implementation is a Taylor-Petrov-Galerkin/pressure-correction scheme that involves a finite element time-stepping technique [18], in conjunction with a recovery scheme to capture continuous velocity gradients. Both decoupled and coupled numerical approaches have been employed previously for a single mode analysis [8], where the results were found to be comparable. Here, only coupled solutions are considered for single-mode computations. For efficiency and pragmatism, multi-mode calculations are performed via a decoupled approach. This strategy is supported by the fact that in many flows the kinematics themselves do not vary significantly with variation of material [19], and hence a reasonable approximation is a linearisation of the system, adopting frozen coefficients and Picard iteration. This implies segmenting the equations of the complete system into those for stress and kinematics separately, computing each to a steady state with frozen coefficients. We note here that the choice of initial frozen kinematic fields are taken as associated with viscoelastic and shear thinning behaviour. Such a pragmatic approach is adopted similarly by others (see Schoonen et al. [20], but with fixed inelastic kinematics).

Single mode modelling is pragmatic, recognised as qualitative not quantitative, and yet effective in terms of relatively low computational cost. A flowrate-maximum shear rate relationship may be established for a particular material and flow, such as within a contraction 
flow. Once this behaviour has been determined, such knowledge may be employed to seek an acceptable rheometrical parameter fit within a neighbourhood of the maximum shear rate, referencing standard viscometric flows. This model specification may then be utilised to predict behaviour in a more general complex flow, such as in a wire-coating setting. To satisfy such criteria, single-mode modelling unfortunately leads to continually having to adjust parameter fits to suit each different flow problem (with flowrate). In this regard, preference shifts to the multi-mode scenario. In contrast, multi-mode modelling with single choice of rheological parameters provides a closer match to the shear viscosity data over a range of shear rates. This facilitates consistent and wide ranging application for complex flows without parameter adjustment. In addition it is possible in the multi-mode context, to identify the contribution of each mode to the total stress and hence determine the most dominant component.

We commence with a single-mode analysis for a Phan-Thien/Tanner (PTT) model and consider the fitting of this model to the rheometrical data of Walters et al. [21], supplied for two independent case studies. The first is essentially a parameter fitting study for viscometric flows, steady simple shear and pure extension. Fitting of the various material parameters $(\epsilon$, $\xi$ and $\mu_{1}$ ) is investigated for shear viscosity in shear flow. Likewise, elongational viscosity behaviour is charted under steady uniaxial extension, according to a Binding analysis for contraction flow [22]. The second case study, involves simulations for a more complex flow, the contraction flow [21]. Here, selected combinations of material parameters, that represent different fluids recommended from the first case study, is employed at different flowrate settings. Of course, ideally one requires a model that for one set of parameters approximates flow over a wide range of conditions. The single mode case proves inadequate for this task. Quantitative agreement is sought on predicted pressure-drop against the available experimental pressure-drop/flowrate data.

The ultimate objective of this work is to relate the significance and sensitivity of the match for the chosen constitutive model to the flow response of the polymer melts in question, under tube-tooling processing conditions. Proceeding in a structured manner from the preliminary case studies above, a single-mode analysis is conducted for this complex industrial flow. Particular attention is paid to pressure-drop and stress build-up across the flow from entry to exit. A second aspect to this work carries over to a multi-mode analysis, under which the three problem settings outlined above are revisited, see [13, 23] for motivation. From a single-mode analysis it is observed that pressure-drop prediction is sensitive to the determination of the material parameters of the PTT model. These are governed by the maximum shear rate sustained in the process at any specific flowrate. Nevertheless, for the complex industrial flows of interest, that involves flow through a tube-tooling die and drawdown section, experimental data is unavailable. Hence for guidance, we turn to a comparison between single and multi-mode model simulations to provide greater insight on the underlying flow behaviour of principle significance.

\section{GOVERNING EQUATIONS AND CONSTITUTIVE MODEL}

The flow of shear and elongational thinning LDPE and HDPE fluids is modelled using a multi-mode Phan-Thien/Tanner (PTT) model, invoking both single and multi-mode approximations. The momentum and continuity equations are given as

$$
\begin{gathered}
\rho \frac{\partial \boldsymbol{u}}{\partial t}=\nabla \cdot \boldsymbol{\tau}-\rho \boldsymbol{u} \cdot \nabla \boldsymbol{u}-\nabla p, \\
\nabla \cdot \boldsymbol{u}=0,
\end{gathered}
$$


with velocity vector $\boldsymbol{u}$, fluid density $\rho$, pressure $p$, stress $\boldsymbol{\tau}$, time $t$ and rate of deformation tensor $\boldsymbol{D}=\left(\nabla \boldsymbol{u}+\nabla \boldsymbol{u}^{\dagger}\right) / 2$. Within the single mode approximation, the stress $\boldsymbol{\tau}$ is decomposed into two parts, viscous and polymeric parts viz

$$
\boldsymbol{\tau}=\boldsymbol{\tau}_{1}+2 \mu_{2} \boldsymbol{D},
$$

for which $\mu_{2}$ is a solvent viscosity. The extra stress tensor $\boldsymbol{\tau}_{1}$ is then defined by

$$
\begin{gathered}
f \boldsymbol{\tau}_{1}+\lambda_{1}{\stackrel{\square}{\boldsymbol{\tau}_{1}}}^{\prime}=2 \mu_{1} \boldsymbol{D}, \\
\stackrel{\square}{\boldsymbol{\tau}}_{1}=\left(1-\frac{\xi}{2}\right) \stackrel{\nabla}{\boldsymbol{\tau}_{1}}+\frac{\xi}{2} \stackrel{\boldsymbol{\tau}}{1}_{1}=\stackrel{\nabla}{\boldsymbol{\tau}_{1}}+\xi\left(\boldsymbol{D} \cdot \boldsymbol{\tau}_{1}+\boldsymbol{\tau}_{1} \cdot \boldsymbol{D}\right),
\end{gathered}
$$

where $f$ is given by

$$
f=\exp \left[\frac{\epsilon \lambda_{1}}{\mu_{1}} \operatorname{trace}\left(\boldsymbol{\tau}_{1}\right)\right],
$$

see $[16,24]$. Material parameters $\lambda_{1}$ and $\mu_{1}$, represent relaxation time and polymeric viscosity, respectively. The zero shear viscosity is then

$$
\mu_{0}=\mu_{1}+\mu_{2} .
$$

Model parameters $(\epsilon \geq 0),(0 \leq \xi \leq 2)$ are non-dimensional parameters that can be evaluated by fitting to experimental data. $\nabla$ and $\Delta$ are upper and lower-convected derivatives, whose combination introduces second normal stress effects.

The PTT model has been chosen because of its shear thinning and strain softening properties, as displayed by the melts in question. Within the complex flows of interest both shear and extension are present. It is instructive, therefore, to analyse the PTT model response in pure shear and extension in isolation. With this in mind, we first present the theoretical response of this model in ideal flows, for which we are able to conduct a multi-variate sensitivity analysis in $\left(\epsilon, \xi, \mu_{1}\right)$ against experimental values of shear viscosity $\mu_{s}$ and extensional viscosity $\mu_{e}$ given by Walters et al. [21]. It is found appropriate to first seek an optimal fit to the shear viscosity, prior to qualifying goodness of fit to extensional viscosity, see below.

In the multi-mode context, the equations of state are given for each mode $(i)$ as

$$
f_{i} \boldsymbol{\tau}_{i}+\lambda_{1}^{i} \stackrel{\square}{\boldsymbol{\tau}_{i}}=2 \mu_{1}^{i} \boldsymbol{D}
$$

where the total stress is

$$
\boldsymbol{\tau}=\sum_{i} \boldsymbol{\tau}_{i}
$$

and $f_{i}$ is defined as above, but with respect to each mode $(i)$. Accordingly, $\lambda_{1}^{i}$ represents the relaxation time of each mode. A vanishing solvent viscosity is considered in the multi-mode case i.e., $\mu_{2}=0$ appropriate for polymer melts, so that here the partial zero-shear viscosity for each mode $(i)$ is $\mu_{0}^{i}=\mu_{1}^{i}$. In this case, $\epsilon$ is the only parameter that is varied, as $\xi$ is chosen to be zero, see on. Hence Eqs. 8 and 9 are solved with Eq. 1 and 2 for momentum and continuity.

In completely general form, retaining a solvent contribution we consider a steady simple shear flow with shear rate $\dot{\gamma}$. Following [25], the representation of the total shear viscosity $\mu_{s}$ over all modes is the sum of the partial shear viscosities, viz

$$
\mu_{s}(\dot{\gamma})=\mu_{2}+\sum_{i} \frac{\mu_{1}^{i} f_{i}}{f_{i}^{2}+\left(\lambda_{1}^{i} \dot{\gamma}\right)^{2} \xi(2-\xi)}
$$


where each $f_{i}$ satisfies

$$
\left\{f_{i}^{2}+\left(\lambda_{1}^{i} \dot{\gamma}\right)^{2} \xi(2-\xi)\right\} \ln \left(f_{i}\right)-2 \epsilon\left(\lambda_{1}^{i} \dot{\gamma}\right)^{2}(1-\xi)=0
$$

Accordingly, first and second normal stress-differences, $N_{1}$ and $N_{2}$ respectively, are defined as

$$
\begin{gathered}
N_{1}(\dot{\gamma})=\sum_{i} \frac{2 \mu_{1}^{i} \lambda_{1}^{i} \dot{\gamma}^{2}}{f_{i}^{2}+\left(\lambda_{1}^{i} \dot{\gamma}\right)^{2} \xi(2-\xi)}, \\
N_{2}(\dot{\gamma})=-\frac{\xi}{2} N_{1}(\dot{\gamma}) .
\end{gathered}
$$

In contrast, for a single mode approximation, the summation collapses to a single term.

In steady uniaxial extension, the elongational viscosity $\mu_{e}$ is a function of extension rate $\dot{\epsilon}$, of the form

$$
\mu_{e}(\dot{\epsilon})=3 \mu_{2}+\sum_{i} \frac{2 \mu_{1}^{i}}{f_{i}^{2}-2 \lambda_{1}^{i} \dot{\epsilon}(1-\xi)}+\frac{\mu_{1}^{i}}{f_{i}+\lambda_{1}^{i} \dot{\epsilon}(1-\xi)}
$$

where each $f_{i}$ is now determined from

$$
\left\{f_{i}^{2}-\lambda_{1}^{i} \dot{\epsilon}(1-\xi)-2\left(\lambda_{1}^{i} \dot{\epsilon}\right)^{2}(1-\xi)^{2}\right\} \ln \left(f_{i}\right)-6\left(\lambda_{1}^{i} \dot{\epsilon}\right)^{2} \epsilon(1-\xi)=0 .
$$

Equations (11) and (15) are solved in each case for the relevant $f_{i}$ through a Newton-Raphson iteration, from which $\mu_{s}, \mu_{e}$, and $N_{1}$ may be determined. We note that via Eq. 13 the second normal stress-difference, $N_{2}$, may be expressed via dependency on $\xi$ and $N_{1}$. In this form, it may be observed that as $\xi$ tends to zero then so does $N_{2}$. Hence, in the multi-mode context, where $\xi$ is taken as zero for simplicity, this implies vanishing $N_{2}$, which in turn justifies the setting of $\mu_{2}=0$.

\section{NUMERICAL SCHEME}

Single-mode solutions are generated employing a coupled procedure previously described in detail in [18]. The coupled scheme involves solving for kinematics and stress simultaneously. The implementation is a fractional-stage time stepping scheme, woven around a pressurecorrection method, that involves some three stages within each time step. The first stage solves for velocity and stress, in a predictor-corrector doublet. The second stage, solves for a pressure temporal increment. The third stage computes a correction to the velocity field, enforcing incompressibility at each time step to the order of the scheme. The scheme embodies implicit and explicit treatment simultaneously, and hence is of classical semi-implicit type. Diffusion terms are approximated with a Crank Nicolson discretisation over a time step, which introduces implicitness and stability for these viscous flows. A direct method of solution is employed to solve for the pressure equation step, whilst indirect Jacobi iteration is invoked for the remaining stages. Also, the benefits are realised of stability enhancing recovery-based methods and consistent streamline upwinding procedures, that are incorporated within the implementation $[26,18]$. For these single-mode coupled calculations a continuation procedure in relaxation time parameter is employed to reach a specific value of $\lambda_{1}^{\text {spec }}$, chosen appropriately, (see on for discussion).

With a multi-mode decoupled procedure, a frozen kinematic field corresponding to a single-mode solution $\left(\lambda_{1}^{\text {spec }}\right)$ is used in Eq. (8) to solve for stress components and each mode $(i)$ of the PTT model in parallel, corresponding to $\left(\lambda_{1}^{i}, \mu_{1}^{i}\right)$ parameters. For simplification, in the multi-mode context, the solvent part of the viscosity $\mu_{2}=0$, and hence for the polymeric part of each mode $(i), \mu_{1}^{i}$ may be replaced by the partial zero-shear rate viscosity $\mu_{i}$, and the 
relaxation time $\lambda_{1}^{i}$ by $\lambda_{i}$. Non-dimensional numbers are defined as follows. For each mode $(i)$, a Weissenberg number is

$$
W e^{i}=\lambda_{i} \frac{U_{c h}}{L_{c h}},
$$

where, $U_{c h}, L_{c h}$ are velocity and length scales, respectively. An average single mode $\lambda_{1}^{\text {spec }}$ is estimated from the experimental data following the procedures outlined in Ref. [27]. This value is gathered from the base material function fits to satisfy $\mu_{s}, \mu_{e}, N_{1}$. For the LDPE polymer, $\lambda_{1}^{\text {spec }}$ is taken as $5 s$, giving a Weissenberg number of $W e^{\text {spec }}$ of 28 according to Eq. 16. Similarly, $\lambda_{1}^{\text {spec }}$ is $9 s$ for HDPE polymer, from which $W e^{\text {spec }}$ equals 50 .

The total zero-shear viscosity is given viz

$$
\mu_{0}=\sum_{i} \mu_{i}
$$

Also, the shear elastic modulus $g_{i}$ for each mode $(i)$, is defined as

$$
g_{i}=\frac{\mu_{i}}{\lambda_{i}}
$$

Time steps of $\mathrm{O}\left(10^{-3}-10^{-4}\right)$ are used and convergence to a steady state is monitored via a relative temporal increment norm on the solution taken to a tolerance of $\mathrm{O}\left(10^{-6}\right)$. A summary of the steps in the numerical procedure is provided as follows:

Stage 1: calculate a Newtonian field $(\boldsymbol{u}, p)$, as in Refs. [28].

Stage 2: Starting from the Newtonian solution and quiescent initial stress conditions a single-mode viscoelastic solution is calculated for $\lambda_{1}^{\text {spec }}$. The Recovery coupled scheme is used with a solvent viscosity of $\mu_{2}=0.01 \mu_{0}$. Thus, viscoelastic kinematics are derived.

Stage 3: Commencing from such a single-mode solution, each of the three stress modes is calculated in a decoupled sense, on resetting the solvent viscosity to zero and freezing the viscoelastic kinematics. For each mode $(i)$, the viscosity, pressure and stress are nondimensionalised as follows

$$
\left(\mu^{i}\right)^{\star}=\frac{\mu^{i}}{\mu_{0}^{i}}=1, p=\mu_{i} \frac{U_{c h}}{L_{c h}} p^{\star} \text { and } \boldsymbol{\tau}_{i}=\mu_{i} \frac{U_{c h}}{L_{c h}} \boldsymbol{\tau}_{i}^{\star}
$$

Stage 4: The total stress is then calculated through Eq. 9. Updated velocity and pressure fields are recomputed by freezing the total stress. Here nondimensionalisation is performed according to the total zero-shear viscosity $\mu_{0}$ given by Eq. 17. After a single pass, this procedure is noted to correct the pressure field, meanwhile variation in the velocity field is found to be insignificant, so that further computation is found subsequently to be unnecessary.

\section{PARAMETER FITTING FOR STEADY SHEAR AND PURE EXTENSION}

\subsection{Single Mode analysis}

Under a single mode analysis, which can be taken as qualitative only, we consider the case of an LDPE polymer. The investigation into parameter sensitivity commences with the polymeric viscosity coefficient $\mu_{1}$. The zero shear viscosity is estimated as $54,700 \mathrm{~Pa}$.s. Employing different fits to the experimental shear data, a single mode relaxation time is evaluated as $\lambda_{1}$ of $5 s$ for LDPE, as cited above (see also $[7,8,21]$. The density $\rho$ for both polymers considered is $760 \mathrm{Kg} / \mathrm{m}^{3}$. 


\subsection{1 $\mu_{1}$ variation}

Theoretically in steady shear flow, by increasing the parameter $\mu_{1}$ and keeping $(\epsilon, \xi)$ fixed, the PTT model thins at high shear rates and accordingly, will give rise to diminishing pressuredrop due to decreasing flow resistance. When $\mu_{1}=0$, there is no polymeric contribution. In Figure 2a, we indicate that the $\mu_{1}$ parameter (recorded in non-dimensional form) plays an important role when the range of the shear rates is high. As the shear rates observed experimentally [21] increase, it is necessary to adjust $\mu_{1}$ to match the data for any isolated shear rate extrema. The inadequacy of a fixed parameter is clearly apparent here in contrast to the multi-mode model (see on). Experimental data were measured for shear viscosity at shear rates between $0.1 s^{-1}$ to $1 s^{-1}$ and $10 s^{-1}$ to $10^{3} s^{-1}$. On the other hand in steady uniaxial extension, the extensional viscosity for the LDPE fluid exhibits strain-softening at high strain-rates, as illustrated in Figure 2b. Such materials may display some hardening at low strain-rates. The PTT model displays a slight increase of the extensional viscosity at extension rates less than unity, before decreasing to a high strain rate limiting plateau. The experimental measurements for elongational viscosity were taken between strain rates of $10 s^{-1}$ and $10^{3} \mathrm{~s}^{-1}$. We comment that the Binding Analysis, used here to derive the experimental $\mu_{e}$ data, is an approximate theory $[22,29,30,31]$. The only difference observed in the fit for different settings of $\mu_{1}$ is in the tail of the curve at high strain rates. As in Figure 2, larger values of $\mu_{1}$ that asymptote to unity, tend to inherit viscosity behaviour of lesser $\mu_{1}$ values over lower shear rate ranges. However, it is necessary to gradually increment values of $\mu_{1}$ towards unity for reasons of numerical convergence in the continuation procedure.

\subsection{2 $\epsilon$ variation}

We proceed to analyse the effect of increasing $\epsilon$ at fixed $\left(\xi, \mu_{1}\right)$. In steady shear flow, the viscosity of the PTT model decreases correspondingly, but at a lower rate than with increase of $\mu_{1}$. Shear thinning behaviour of this form, with increasing $\epsilon$, will generate a decrease in pressure-drop. In Figure 3a with $\epsilon=0.15$, the PTT model provides a near-optimal least squares fit across the complete range of shear rates displayed, for a match to the experimental data at a maximum value of $\dot{\gamma}=10^{3} \mathrm{~s}^{-1}$. In contrast, a best fit for shear rates less than $10 \mathrm{~s}^{-1}$ is for a value of $\epsilon=0.7$. Switching to uniaxial extension, a reduction in elongational viscosity with increase in $\epsilon$ is observed, yet without influencing the asymptotic plateau at high strain rates. Clearly, here there is only limited experimental data available and the model can only give a qualitative representation of elongational behaviour.

\subsection{3 $\xi$ variation}

Finally, adjusting the parameter $\xi$ for constant values of $\mu_{1}$ and $\epsilon$, gives a decrease in the shear viscosity for increasing $\xi$. At high values of $\epsilon=1.0$, there is barely any change in the shear viscosity for $0 \leq \xi \leq 0.1$. Some departure from the experimental values is noted at the lower value of $\epsilon=0.15$ for $\xi \geq 0.1$. The elongational viscosity is unaffected by such minor adjustment in parameter $\xi$, that is with the exception of $\xi=1$, for which a constant elongational viscosity is derived. The comment above regarding quality of representation also applies here.

\subsection{Multi-mode analysis}

Table 1 provides material data for LDPE and HDPE polymers at a temperature of $200^{\circ} \mathrm{C}$ [32], in the form of relaxation times and partial zero-shear rate viscosities for a three-mode 
approximation. The fits to the the shear data yield zero-shear viscosities of 105,390 and 139,184 Pa.s for LDPE and HDPE, respectively. Note, the variation to the single-mode case is due to the difference in parameters that alters the fits. Any solvent viscosity contribution is taken as minuscule for the polymer melts of interest, simply a mathematical convenience to both aid data fitting and numerical convergence. For a melt any solvent contribution is insignificant in practice; hence in the multi-mode context, $\mu_{2}$ is assumed to vanish. Here, this is made practically possible from a numerical standpoint via the decoupled approach employed. Also, the second normal stress difference $\left(N_{2}\right)$ is negligible compared with the first normal stress difference $\left(N_{1}\right)$. Hence from Eq. 13, the value of the non-dimensional parameter $\xi$ is small, and to simplify the analysis can be taken as zero. To estimate the remaining parameter $\epsilon$, the shear viscosity is plotted against the shear rate in Figure 3a for LDPE and Figure 4a for HDPE with various values of $\epsilon$. For both materials, the best fit for $10^{2} \leq \dot{\gamma} \leq 10^{3} \mathrm{~s}^{-1}$ corresponds to a value of $\epsilon$ of unity. At such a value, we observe from both Figures $3 \mathrm{~b}$ and $4 \mathrm{~b}$, the elongational viscosity $\left(\mu_{e}\right)$ fits provide appropriate trends in general behaviour, but gives a lower estimated value than the limited set of experimental data. This is due to the inability of the PTT model to match both shear and elongational data simultaneously.

From the knowledge gained through the single-mode study, we observe that the key factor to estimating the pressure-drop accurately in complex flows (see below) is the quality of fit to the shear viscosity 'in situ' at the associated maxima of shear rate. Bearing this point in mind, in Figure 3 for LDPE we compare shear and elongational viscosity fits to the experimental data for both the single mode of $\lambda_{1}=5 s$ (from above) and a three-mode model. In general, and accordance with Figure 3 a for shear viscosity, the multi-mode case provides the better fit to the experimental data, throughout a wide range of shear rate. The multi-mode trends of response for elongational viscosity of Figure $3 \mathrm{~b}$ is a much better reflection of the actual fluid properties than those observed with a single-mode approximation. Nevertheless, the match here is somewhat adrift from the experimental data values, derived from the Binding analysis. The same fitting procedure has also been carried out using a seven mode model. It is of note that no substantial improvement of fit is obtained if seven modes are employed in place of three (see Matallah et al. [15]).

\section{CONTRACTION FLOW}

Armed with the parameter fits to steady shear and pure extension, we proceed to analyse a contraction flow, testing our simulated solution pressure-drops across the domain against those observed experimentally at a series of different flowrates [21].

\subsection{Problem specification}

A schematic flow diagram for the axi-symmetric 19:1 contraction flow with its associated finite element mesh is represented in Figure 5. The finite element discretisation is represented by 1830 elements and 3829 nodes. This problem is representative of flow in a contraction Capillary rheometer. The flow may be assumed to be symmetric about the central axis. Characteristic length and velocity scales are taken as the radius $R_{c}$ of the die tube and mean exit velocity $U_{c}$, whilst the zero-shear rate viscosity $\mu_{0}$ is considered as the characteristic viscosity. A non-dimensional Reynolds number is defined as

$$
R e=\frac{\rho U_{c} R_{c}}{\mu_{0}}
$$


and, for a single relaxation time, a Weissenberg number is taken as

$$
W e=\lambda_{1}^{\text {spec }} \frac{U_{c}}{R_{c}} .
$$

For the multi-mode case, see above for comparable definitions. With respect to boundary conditions, no-slip conditions are taken on the downstream tube walls, and symmetry conditions apply on the flow centreline. The upstream length is chosen to be sufficiently large $68 R_{c}$, to establish fully developed Poiseuille flow in the inlet region. The downstream die length is $40 R_{c}$. At the domain exit, a fixed pressure datum is adopted for consistency and a fully developed Poiseuille flow prevails. Each individual flowrate is treated as a separate problem to which a steady state is sought. Though we are interested in steady state solutions, we note that these complex flows are transient in Lagrangian sense, so that a particle following the flow will in fact encounter different conditions at different locations (or times). For a single-mode, initial conditions are taken of a Newtonian kinematic field, coupled to a fully relaxed stress field with appropriate inlet boundary modification. In the multi-mode case, initial kinematics are supplied from a prior single-mode computation. Then for each stress component mode, it is found suitable to impose relaxed inlet boundary conditions that rapidly adopt their appropriate levels in the entry flow region.

\subsection{Results for a single mode model}

In this section, we analyse our results in trends only for single-mode modelling. To establish a pressure drop versus flowrate relationship numerically and to compare this to experimental observations, three flowrates have been employed for different PTT fluid parameter sets $(\epsilon, \xi)$, in combination with $\mu_{1}$. A given flowrate, $Q$, covers a particular shear-rate range. The shear viscosity $\mu_{s}(\dot{\gamma})$ may be adjusted through $\mu_{1}, \epsilon$ and $\xi$ parameters to fit the experimental data. Indeed, rising $\mu_{1}$ tending towards $\mu_{0}$, governs the second Newtonian plateau level for $\mu_{s}(\dot{\gamma})$. Hence, elevating $\mu_{1}$, effectively extends the range of fit for the $\mu_{s}(\dot{\gamma})$ function over wider $\dot{\gamma}$ ranges. Thus the larger the value of $Q$, the more $\mu_{1}$ must be elevated to enhance the $\mu_{s}(\dot{\gamma})$ fit to the data. The classification of various test fluids, with $\mu_{1}$-values rising up to $\mu_{0}$, highlights Fluids B-H as indicated in Table 2. There, $\mu_{1}=0.875 \mu_{0}$ for Fluid B, $\mu_{1}=0.99 \mu_{0}$ for Fluid C, $\mu_{1}=0.995 \mu_{0}$ for Fluid D, $\mu_{1}=0.999 \mu_{0}$ for Fluid E, $\mu_{1}=0.998 \mu_{0}$ for Fluid F and $\mu_{1}=\mu_{0}$ for Fluids $\mathrm{G}$ and $\mathrm{H}$. We note that, lower $\mu_{1}$-values aids numerical convergence as the solvent contribution incorporates damping into the system. For the single mode approximation, a single relaxation time of $\lambda_{1}^{\text {spec }}=5 \mathrm{~s}$ is adopted to represent the relaxation time spectrum for an LDPE polymer (see Ref. [32]). Reynolds numbers are of $\mathrm{O}\left(10^{-6}\right)$, whilst Weissenberg number values at various flowrates are summarised in Table 3 , and pressure-drop results in Table 4 and Figure 6.

Overall, a summary of our findings reads as follows. By comparing the numerical and experimental data for these three flowrates, and investigating the parameter sensitivity of shear viscosity on $\mu_{1}, \epsilon$ and $\xi$ at different shear rates, we conclude that the most influential parameter governing $\Delta p$ is $\mu_{1}$; the remaining parameters $(\epsilon, \xi)$ provide fine tuning of the fit. The choice of parameter $\mu_{1}$ depends on the value of maximum shear rate $\dot{\gamma}_{\text {max }}$ attained for any given flowrate. Hence with knowledge of $\dot{\gamma}_{\max }, \mu_{1}$ may be determined by choosing a value to fit the experimental data of [21]. In Figure 7, maximum shear rates observed for the contraction flow are plotted against flowrate, from which a quadratic relationship emerges. From this, one may predict $\dot{\gamma}_{\max }$ for a given flowrate and hence extract a particular $\mu_{1}$-fit (Figure 6).

It is clear that with a single-mode approximation, it is necessary to continually adjust the fluid parameters at each flowrate setting, to adequately reproduce quantitative pressure-drop 
predictions. This keeps pace with the shear rate maxima observed, though does not provide a single fluid model representation suitable across a range of flowrates. This is a drawback to the use of a single mode PTT model. Essentially, what is being achieved here is to provide a localised fit to data around the range of shear rates that dominates the process at each particular flowrate. As regards incrementation of $\mu_{1}$ towards unity, this leads to more severe numerical difficulties in convergence to steady state. Thus care in continuation with this parameter is advisable purely on numerical grounds. At this juncture, it is natural to widen the study to embrace a multi-mode approximation and follow a similar line of investigation.

\subsection{Results for a three-mode model}

With a three-mode PTT approximation, results for both LDPE and HDPE grade polymers are presented and compared to the experimental data for the contraction flow. Comparison against the single-mode approximation is made only for the LDPE polymer, and $\xi$ is taken as zero (see above, $N_{2}<<N_{1}$ ). Our observations are that computation times double from single to three-mode model calculations.

\subsubsection{LDPE fluid}

With the LDPE polymer a first flowrate of $Q_{2}=39.9 \mathrm{~mm}^{3} / \mathrm{s}$ is considered, for which the values of Weissenberg numbers for the corresponding three modes are given in Table 3 . The Reynolds number is $R e=1.8 * 10^{-7}$ and the experimental pressure-drop is $10.9 \mathrm{MPa}$. With fluid $G_{1}$ offering a parameter combination $\left(\epsilon, \xi, \mu_{1}\right)=\left(1.0,0.0, \mu_{0}\right)$, a simulated pressure-drop of $10.3 \mathrm{MPa}$ is predicted. This contrasts to the best single mode computation of $11.7 \mathrm{MPa}$ for fluid $D_{2}$ with $\left(\epsilon, \xi, \mu_{1}\right)=\left(0.3,0.02,0.995 \mu_{0}\right)$.

In contrast, at a second lesser flowrate of $Q_{1}=2.37 \mathrm{~mm}^{3} / \mathrm{s}$, and the same parameter combination $\left(\epsilon, \xi, \mu_{1}\right)=\left(1.0,0.0, \mu_{0}\right)$, i.e. fluid $G_{1}$, a simulated pressure-drop of $5.90 \mathrm{MPa}$ is generated; an over-estimation compared to the experimental data of $3.27 \mathrm{MPa}$. This is in accordance with the shear viscosity fit, where for the shear rate range $10 \leq \dot{\gamma} \leq 10^{2} s^{-1}$, the best fit renders a value of $\epsilon=2.5$. For fluid $G_{2}$ of $\epsilon=2.5$, the pressure-drop is $4.00 \mathrm{MPa}$ compared to $4.09 \mathrm{MPa}$ for the single mode calculation of fluid $C_{1}$. Figure 8 a summarises graphically the comparison of numerical and experimental data at both flowrates, including the single mode results. The values are tabulated in a unified manner in Table 4. Conventional use of the PTT class of models, is restrictive with a constant value for the parameter $\epsilon$. If this parameter is taken in the range $1.0 \leq \epsilon \leq 2.5$, pressure-drop will be better estimated by the multi-mode than the single mode model for the flowrate range $2.37 \leq Q \leq 40 \mathrm{~mm}^{3} / \mathrm{s}$. We observe in Figure 8a, that the discrepancy from the experimental value is huge for the single mode fluid $C_{1}$ - parameter set $\left(\epsilon, \xi, \mu_{1}\right)=\left(0.15,0.02,0.99 \mu_{0}\right)$ at $Q_{2}=39.9 \mathrm{~mm}^{3} / \mathrm{s}$.

\subsubsection{HDPE fluid}

For the HDPE polymer, computations are conducted for flowrates of $Q_{1}=2.37 \mathrm{~mm}^{3} / \mathrm{s}$ and $Q_{2}=34.0 \mathrm{~mm}^{3} / \mathrm{s}$. At the former flowrate, the fitting of the PTT model to the experimental shear viscosity, as in Figure 4, provides an equally good dual set of parameters with either $(\epsilon$, $\left.\xi, \mu_{1}\right)=\left(2.5,0.0, \mu_{0}\right)$ or $\left(\epsilon, \xi, \mu_{1}\right)=\left(3.0,0.0, \mu_{0}\right)$, i.e. fluid $H_{2}$ or $H_{3}$. Simulated pressure-drops are 7.40 MPa for fluid $H_{2} \epsilon=2.5$, and 6.90MPa for fluid $H_{3} \epsilon=3.0$, that compare against an experimental value of $\Delta p=5.83 \mathrm{MPa}$. It is implied that additional increase in the parameter $\epsilon$, will lead to further decrease in pressure-drop.

At a second flowrate of $Q_{2}=34.0 \mathrm{~mm}^{3} / \mathrm{s}$, and according to Figure 4 , the best fit to the shear viscosity data, results in the set of parameters $\left(\epsilon, \xi, \mu_{1}\right)=\left(1.0,0.0, \mu_{0}\right)$ for the 
range $10^{2} \leq \dot{\gamma} \leq 10^{3} \mathrm{~s}^{-1}$, i.e. fluid $H_{1}$. The simulated pressure-drop is $19.2 \mathrm{MPa}$, whilst the experimental value is $19.9 \mathrm{MPa}$. This evidence is taken as endorsement for the choice of multimode representation with fluids $G_{1}$ and $H_{1}$, that is wholy acceptable across the flowrates selected. A summary of simulated and experimental pressure-drop values is provided for HDPE in Table 5 and Figure 8b.

\section{WIRE-COATING FLOW}

Having established the goodness of fit to experimental pressure-drop data for a complex contraction flow, we now proceed to the industrial flow of interest that provides the motivation for this study. The flow quantities of specific relevance are residual flow-induced stress and pressure-drop. The former is fresh information to be gathered for this problem that is important to control coating properties. These quantities have impact on the optimisation of the process design. Only fluid $G_{1}$ (i.e. one set of parameters $(\epsilon, \xi)=(1,0)$ ) is adopted throughout the study of tube-tooling wire-coating for LDPE and $H_{1}$ for HDPE polymer, since a reasonable fit is achieved in the shear-rate range under consideration $\left(\dot{\gamma} \leq 10^{3} s^{-1}\right)$.

\subsection{Problem specification}

A schematic flow diagram for the tube-tooling problem with its finite element mesh is displayed in Figure 9. The flow enters the annular tube AB, then a converging cone section $\mathrm{BC}$ and a land region $\mathrm{CD}$, and is draw-down by the wire in a converging cone form $\mathrm{DE}$, and coating part of the wire EF, as shown in Figure 9a. Due to symmetry, it is necessary to model only one half of the problem. Characteristic length and velocity scales are taken as the horizontal distance between the die exit of the tube and the contact point on the wire, i.e., the draw-down length $L_{d r a w}$ and the velocity of the wire $U_{\text {wire }}$, respectively. The zeroshear viscosity $\mu_{0}$ is considered as the characteristic viscosity. Here, the same definition for Reynolds and Weissenberg numbers is taken as for the contraction flow problem. Boundary conditions are given as follows. No-slip conditions are taken on tube walls for the die tube, ABCD and D'C'B'A'. At the entry AA' a fully developed Newtonian annular velocity profile corresponding to a specific flow rate $Q$ is imposed, $\left(U_{r}=0\right.$ and $\left.V_{z}=V_{z}(r)\right)$. From this velocity profile, a PTT stress profile is generated analytically. This specifies inlet flow boundary conditions. Free surface boundary conditions along DE and F'D' are taken equivalent to the average velocity per cross sectional area, consistent with draw-down flow. On EF, the fluid is considered to be moving with the wire (no slip). A uniform structured mesh is used in the finite element discretisation, 2680 elements and 5649 nodes, (thoroughly investigated in our prior studies, see Mutlu et al. [8,9]). To capture sharp velocity gradients near singular regions, different meshes were employed elsewhere (see $[8,33]$ ). In the present study only the finest mesh of Ref. [8] is employed, for which the number of elements per region is given in Table 6 .

\subsection{LDPE fluid}

Table 7 provides values of Weissenberg numbers for an LDPE polymer, with material parameters given in Table 1, for a standard flowrate (1Q) and double flowrate (2Q). The Reynolds numbers are $R e=1.5 * 10^{-4}$ and $R e=3.0 * 10^{-4}$, respectively. Figure 10 plots the pressuredrop line for both $1 \mathrm{Q}$ and $2 \mathrm{Q}$, along a sample line tangential to the flow. The sample line for plotting lies along the inner radius of the annular settings. Cross stream variation is hardly significant. Simulated pressure-drop values are 6.90 and $8.40 \mathrm{MPa}$, respectively, and are compared to 7.49 and $11.2 \mathrm{MPa}$ using fluid $C_{1}$ for the single mode approximation (as [8] 
using $\mu_{1}=0.99 \mu_{0}, \epsilon=0.15$ and $\left.\xi=0.02\right)$. Pressure-drop results are tabulated in Table 8 accordingly. For the single mode, pressure-drop at $2 \mathrm{Q}$ is one and a half times that at $1 \mathrm{Q}$, whilst for a multi-mode model, this factor reduces to 1.22. Overall, the trend in variation of pressure-drop is similar for single and multi-mode models in the tube-tooling die sections, notwithstanding the elevation of the single-mode $2 \mathrm{Q}$ result. In contrast in the draw-down section for the single mode, pressure-drop is almost constant for $1 \mathrm{Q}$, but for $2 \mathrm{Q}$, it increases around the location where the fluid meets the wire. Traveling with the wire, pressure diminishes to zero. However for a multi-mode model, the pressure-drop decreases continuously with only a slight change in the slope on encountering the wire. These differences in response for these models, are due to the variation in radial shear stress gradients, particularly at die exit, that are about one third of the level observed in the inlet tube-tooling section.

In Figure 11, the shear stress is plotted along the inner annular radius. A constant value is observed in the inlet tube (shear flow), followed by a change of sign over the contracting flow within the tube-tooling cone. Shear stress increases in absolute value as the cone contracts. In the land section, the degree of shearing increases again, sharply at the start, flattens across the land region, and rises slightly at the end. There is then a dramatic sharp drop and oscillation in shear stress at the inlet of the draw-down cone. This is where there is a sudden adjustment from the annular shear flow to an extensional drawing flow. Thereafter, it decreases gradually but smoothly along the draw-down cone, to increase slightly when the wire is met and subsequently remains steady. This behaviour is similar for both flowrates, only differing in the absolute level of the stress.

For the normal stress component in the axial direction, $\tau_{z z}$, a sharp oscillation is experienced at the inlet of the tube-tooling cone due to the sudden geometry changes. This is followed by a smooth increase in $\tau_{z z}$ as the shear rate increases in the cone. Over the land region, $\tau_{z z}$ suddenly decreases as the polymer enters, and remains fairly constant to the land region exit. A shock is noted in the transition from land to draw-down flow. Over the drawdown section, the stress component decreases. A smaller variation rate in $\tau_{z z}$ is observed as opposed to that in the tube-tooling cone, and there is a marginal increase when the fluid meets the wire. A relaxation of stress occurs within the coating flow on the wire, with larger slope than in the draw-down cone.

In summary, a build-up of shear and normal stress is observed in the tube-tooling cone, followed by relaxation in the draw-down and wire-coating flow sections.

For stress, the trends are identical for single to multi-mode cases, the differences lie in the stress levels, that are dictated by the inlet flow. At inlet and for the shear rates that apply there, see Figure 3, a closer match to $\mu_{s}$ is observed with a single mode model, yielding a lower value of shear stress (via viscosity) than with the multi-mode model. We note also that in the converging tube the rate of increase of $\tau_{z z}$ in the multi-mode case is greater than that corresponding to the single mode instance. This is due to the influence of the shortest mode $\lambda_{1}=0.017 s$; see below for further comment on the contributions due to the separate modes.

\subsection{HDPE fluid}

Results for the HDPE polymer are also presented at the same flowrates as above for LDPE. In this case, the parameter $\epsilon=1.0$ represents the best fit to the shear viscosity, as in Figure 4, hence fluid $H_{1}$. Predicted pressure-drops are 12.4 and $15.4 \mathrm{MPa}$ for the two associated flowrates, respectively. Figure 12 shows a sample line plot for pressure-drop at both flowrates, and a similar trend to that for the LDPE polymer is observed. We note that pressure-drop at the double flowrate is 1.24 times as great as that at the standard flowrate; the factor relating pressure-drops is similar to that found for LDPE, of 1.22. It is conspicuous that 
corresponding pressure-drops for HDPE polymer are almost double those for LDPE polymer. According to Figure 13, line plots for shear and normal stress components along a sample line tangential to the flow, the general behaviour of stress is similar to that for LDPE, though scales are doubled in shear stress and increase by about $20 \%$ in normal stress. These findings are in keeping with general expectations for these materials and flows.

\subsection{Analysis of separate modes}

To understand the contribution of each mode $(i)$ to the total stress of the multi-mode model, both $\tau_{r z}$ and $\tau_{z z}$ are plotted for the standard flowrate, LDPE polymer in Figure 14. Of the individual modal contributions, the two shorter relaxation time modes dominate. The shear stress is dominated by the shortest relaxation time. This is true for all cases studied, covering both materials and flowrates, as confirmed in Figure 14 for LDPE and Figure 15 for HDPE.

Alternatively, for the $\tau_{z z}$ component, according to Figure 14 and LDPE, the shortest relaxation time dominates in the tube-tooling cone; this mode dictates the shape of the total stress in all the sections of the flow. In contrast, the second shortest relaxation time dominates in the inlet tube and draw-down sections, including the flow on the wire. For the HDPE polymer, as in Figure 15, the shortest relaxation time dominates throughout the tube-tooling die sections, whilst in the draw-down, the second shortest relaxation time dominates.

\section{CONCLUSIONS}

A contraction flow has been used effectively to demonstrate how single and multi-mode PTT models perform in quantitatively replicating pressure-drop/flowrate data. Essentially this is governed by the goodness of fit to the shear viscosity. In this regard, the multi-mode instance performs better across the range of shear rates of interest. In the single-mode case reasonable qualitative correspondence has been derived, presupposing a judicious choice of averaged relaxation time, which has been possible here. Also, the choice of material parameter combination must vary with each flowrate (and its associated maximum shear rate) to adequately reflect the experimental data. This generates different fluid model representations and demonstrates the shortcomings of an averaged mode approximation. The multi-mode alternative, performs better in this respect with a fixed fluid parameter set.

In the more generalised context of the industrial tube-tooling wire-coating flow, the differences in results on stress distributions and pressure-drop between these modelling approaches is more stark. Here, the multi-mode approximation reveals the fine detail of stress response throughout the flow, peak values attained and dominance of individual components. The key point emerges that the shorter relaxation time modes are observed to dominate within the process. These modes are of the order of less than one second, being synonymous with a representative particle residence time as it travels through the flow domain. Stress and pressure-drop levels are practically doubled for the HDPE above the LDPE polymer, which is consistent with practical industrial experience for these materials. Also pressure-drop variation with flowrate doubling is held to be more realistic with the multi-mode option. The differences from single to multi-mode total stress appear minimal in the draw-down and coating regions, taking into account the elevations in stress levels within the steady shear die entry flow as discussed above. 


\section{ACKNOWLEDGEMENTS}

The authors wish to gratefully acknowledge the financial support and collaboration of BICC Cables Ltd. Thanks is also due to Professor Ken Walters, FRS, Professor Russell Davies and Dr David Binding for their support work on rheological characterisation.

\section{References}

[1] R.T. Fenner and J.G. Williams. Trans. J. Plastics Inst., pages 701-706, 1967.

[2] B. Caswell and R.I. Tanner. 'Wirecoating die design using finite element methods'. Polymer Eng. Sci., 18 (5):416-421, (1978).

[3] E. Mitsoulis. 'Fluid flow and heat transfer in wire coating: A review'. Advances in Polymer Technology, 6 (4):467-487, (1986).

[4] E. Mitsoulis, R. Wagner, and F.L. Heng. 'Numerical simulation of wire-coating lowdensity polyethylene: Theory and experiments'. Polymer Eng. Sci., 28 (5):291-311, (1988).

[5] H.C. Huang, P. Townsend, and M.F. Webster. Numerical Grid Generation in Computational Fluid Dynamics and Related Fields. N.P. Weatherill and P.R. Eiseman and J. Hauser and J.F. Thompson, Pineridge Press, Swansea, 1994.

[6] D.M. Binding, A.R. Blythe, S. Gunter, A.A. Mosquera, P. Townsend, and M.F. Webster. 'Modelling polymer melt flows in wirecoating processes related fields'. J. Non-Newtonian Fluid Mech., 64:191-206, (1996).

[7] S. Gunter, P. Townsend, and M.F. Webster. 'Simulation of some model viscoelastic extensional flows'. Int. J. Num. Meth. Fluids, 23:691-710, (1996).

[8] I. Mutlu, P. Townsend, and M.F. Webster. 'Simulation of cable-coating viscoelastic flows with coupled and decoupled schemes'. J. Non-Newtonian Fluid Mech., 74:1-23, (1998).

[9] I. Mutlu, P. Townsend, and M.F. Webster. 'Computation of viscoelastic cable coating flows'. Int. J. Numer. Meth. Fluids, 26:697-712, (1998).

[10] I. Mutlu, P. Townsend, and M.F. Webster. 'Mathematics of finite elements and applications-highlights 1996'. ed. J.R. Whiteman, Wiley, Chichester, 18:299-312, (1997).

[11] H.P.W. Baaijens. Evaluation of constitutive equations for polymer melts and solutions in complex flows. PhD. Thesis, Eindhoven University of Technology, Netherlands, 1994.

[12] F.P.T. Baaijens, S.H.A. Selen, H.P.W. Baaijens, G.W.M. Peters, and H.E.H. Meijer. 'Viscoelastic flow past a confined cylinder of a LDPE melt'. J. Non-Newtonian Fluid Mech., 68:173-203, (1997).

[13] J. Azaiez, R. Guénette, and A. Ait-Kadi. 'Entry flow calculations using multi-mode models'. J. Non-Newtonian Fluid Mech., 66:271-281, (1996).

[14] M. Gupta, C.A. Hieber, and K.K. Wang. 'Viscoelastic modelling of entrance flow using multimode Leonov model'. Int. J. Numer. Meth. Fluids, 24:493-517, (1997). 
[15] H. Matallah, P. Townsend, and M.F. Webster. 'Viscoelastic multi-mode simulations of wire-coating'. J. Non-Newtonian Fluid Mech., 90:217-241, (2000).

[16] N. Phan-Thien and R.I. Tanner. 'A new constitutive equation derived from network theory'. J. Non-Newtonian Fluid Mech., 2:353-365, (1977).

[17] P. Saramito and J.M. Piau. 'Flow characteristics of viscoelastic fluids in an abrupt contraction by using numerical modelling'. J. Non-Newtonian Fluid Mech., 52:263-288, (1994).

[18] H. Matallah, P. Townsend, and M.F. Webster. 'Recovery and stress-splitting schemes for viscoelastic flows'. J. Non-Newtonian Fluid Mech., 75:139-166, (1998).

[19] J.R.A. Pearson and S.M. Richardson. Computational Analysis of Polymer Processing. Applied Science Publishers Ltd, London and New York, 1983.

[20] J.F.M. Schoonen, F.H.M. Swartjes, G.W.M. Peters, F.P.T. Baaijens, and H.E.H. Meijer. 'A 3D numerical/experimental study on a stagnation flow of a polyisobutylene solution'. J. Non-Newtonian Fluid Mech., 79:529-561, (1998).

[21] K. Walters, D.M. Binding, and R.E. Evans. 'Modelling the rheometric behaviour of 3 polyethylene melts'. Technical report, Institute of non-Newtonian Fluid Mechanics, University of Wales, Aberystwyth, May 10, 1994.

[22] D.M. Binding. 'An approximate analysis for contraction and converging flows'. J. NonNewtonian Fluid Mech., 27:173-189, (1988).

[23] L.M. Quinzani, G.H. McKinley, R.A. Brown, and R.C. Armstrong. 'Modeling the rheology of polyisobutylene solutions'. J. Rheol., 34:705-748, (1990).

[24] N. Phan-Thien. 'A non-linear network viscoelastic model'. J. Rheol., 22:259-283, (1978).

[25] A. Arsac, C. Carrot, J. Guillet, and P. Revenu. 'Problems originating from the use of the Gordon-Schowalter derivative in the Johnson-Segalman and related models in various shear-flow situations'. J. Non-Newtonian Fluid Mech., 55:21-36, (1994).

[26] O.C. Zienkiewicz and J.Z. Zhu. 'Superconvergence and the superconvergent patch recovery'. Finite Element in Analysis and Design, 19:11-23, (1995).

[27] S. Gunter, M.F. Webster, and P. Townsend. BICC internal report. Computer Science Department, University of Wales, Swansea, 1995.

[28] D.M. Hawken, H.R. Tamaddon-Jahromi, P. Townsend, and M.F. Webster. 'A TaylorGalerkin based algorithm for viscous incompressible flow'. Int. J. Num. Meth. Fluids, 10:327-351, (1990).

[29] F.N. Cogswell. 'Converging flow of polymer melts in extrusion dies'. Polymer Eng. Sci., 12:64-74, (1972).

[30] D.M. Binding. In: A.A. Collyer (Ed.), Techniques in Rheological Measurement. Chapman and Hall, London, UK, Chap 1, 1993.

[31] D.M. Binding, M.A. Couch, and K. Walters. 'The pressure dependence of the shear and elongational properties of polymer melts'. J. of Non-Newtonian Fluid Mech., 79:137$155,(1998)$. 
[32] A.R. Davies, K. Walters, and D.M. Binding. 'An analysis of the discrete relaxation spectra of 3 polyethylene samples for BICC group', private communications. Technical report, Institute of non-Newtonian Fluid Mechanics, University of Wales, Aberystwyth, June 20, 1996.

[33] I. Mutlu, P. Townsend, and M.F. Webster. 'Adaptive solutions for viscoelastic flows'. Commun. Numer. Meth. Eng., 12:643-655, (1996). 


\section{List of Tables}

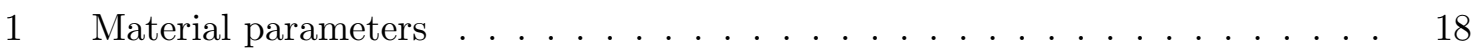

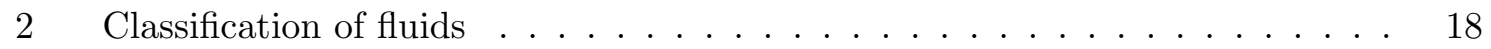

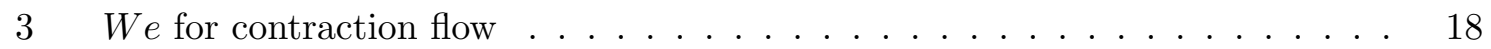

4 Pressure drop for contraction flow, LDPE polymer . . . . . . . . . . . 18

5 Pressure drop for contraction flow, HDPE polymer, multi-mode . . . . . . . 19

6 Number of elements per region . . . . . . . . . . . . . . . . . . . 19

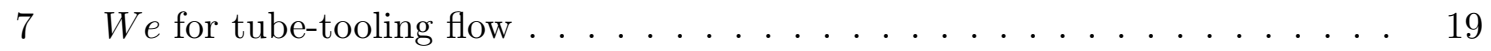

8 Pressure drop for tube-tooling flow . . . . . . . . . . . . . 19

\section{List of Figures}

1 Schematic diagram of wire coating dies: (a) tube-tooling die; (b) pressuretooling die. . . . . . . . . . . . . . . . . . 20

2 Shear and elongational viscosities fits; $\mu_{1}$ variation, $\epsilon=0.15, \xi=0.02 . \ldots 21$

3 Single and multi-mode fits for LDPE. . . . . . . . . . . . . . . . 22

4 Rheometrical data fits with $\epsilon$ variation for 3-mode HDPE. . . . . . . . . . . . 23

5 Schematic flow diagram and finite element mesh for 19:1 contraction geometry. 24

6 Pressure drop v flowrate for contraction problem, single mode model . . . . . 25

$7 \quad$ Maximum shear-rate v flowrate correlation for contraction problem, LDPE . 26

8 Simulated and experimental pressure drops for contraction flow; a) LDPE, b)

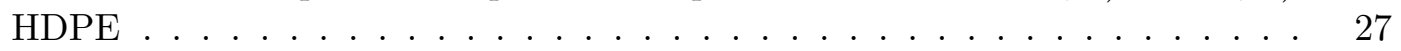

$9 \quad$ Schematic diagram and finite element mesh for wire coating tube-tooling die. 28

10 Tube-tooling flow, streamwise pressure profiles, single and multi-mode models, LDPE . . . . . . . . . . . . . . . . . . . . 29

11 Tube-tooling flow, streamwise stress profiles, single and multi-mode models, LDPE, a) $\tau_{r z}$, b) $\tau_{z z} \ldots \ldots \ldots$. . . . . . . . . . . . . . . . 30

12 Tube-tooling flow, streamwise pressure profiles, standard and double flowrate,

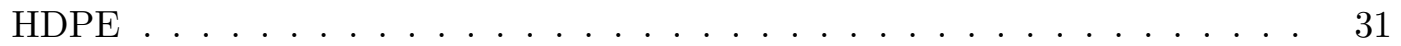

13 Tube-tooling flow, streamwise stress profiles, multi-mode, HDPE, a) $\tau_{r z}$, b) $\tau_{z z} 32$

14 Tube-tooling flow, streamwise stress profiles for individual stress mode, LDPE,

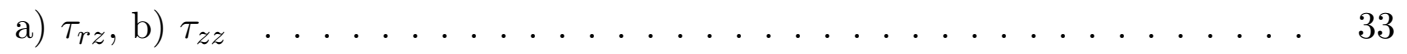

15 Tube-tooling flow, streamwise stress profiles for individual stress mode, HDPE, a) $\tau_{r z}$, b) $\tau_{z z} \ldots \ldots \ldots \ldots \ldots \ldots$ 


\begin{tabular}{|l|ccc|ccc|}
\hline \hline & \multicolumn{3}{|c|}{ LDPE } & \multicolumn{3}{c|}{ HDPE } \\
\hline & $\lambda_{i}(s)$ & $\mu_{i}($ Pa.s $)$ & $g_{i}=\frac{\mu_{i}}{\lambda_{i}}$ & $\lambda_{i}(s)$ & $\mu_{i}($ Pa.s $)$ & $g_{i}=\frac{\mu_{i}}{\lambda_{i}}$ \\
\hline Mode 1 & 0.017 & 1234 & 72588 & 0.017 & 2792 & 164222 \\
Mode 2 & 0.87 & 15982 & 18370 & 0.87 & 19595 & 22523 \\
Mode 3 & 33.9 & 88174 & 2601 & 59.57 & 116797 & 1961 \\
& & & & & & \\
\hline
\end{tabular}

Table 1: Material parameters

\begin{tabular}{|c|c|c|}
\hline Fluid B $\mu_{1}=0.875 \mu_{0}$ & Fluid C $\mu_{1}=0.99 \mu_{0}$ & Fluid $\mathrm{D} \mu_{1}=0.995 \mu_{0}$ \\
\hline$B_{1}:(0.1,0.01)$ & $C_{1}(0.15,0.02)$ & $D_{1}(0.15,0.02)$ \\
$B_{2}:(0.3,0.01)$ & $C_{2}(2.0,0.02)$ & $D_{2}(0.3,0.02)$ \\
$B_{3}:(2.0,0.01)$ & & \\
\hline Fluid $\mathrm{E} \mu_{1}=0.999 \mu_{0}$ & Fluid $\mathrm{F} \mu_{1}=0.998 \mu_{0}$ & Fluid $\mathrm{G} \mu_{1}=\mu_{0}, \xi=0$ \\
$(0.3,0.02)$ & $(0.15,0.02)$ & ---------- \\
& & $G_{1}: \epsilon=1.0$ \\
& & $G_{2}: \epsilon=2.5$ \\
& & $G_{3}: \epsilon=3.0$ \\
\hline & & Fluid $\mathrm{H} \mu_{1}=\mu_{0}, \xi=0$ \\
& & ------------- \\
& & $H_{1}: \epsilon=1.0$ \\
& & $H_{2}: \epsilon=2.5$ \\
& & $H_{3}: \epsilon=3.0$ \\
\hline
\end{tabular}

Table 2: Classification of fluids

\begin{tabular}{|l|c|cc|cc|}
\hline \hline & & LDPE & & HDPE & \\
\hline & $\mathrm{Q} \mathrm{mm}^{3} / \mathrm{s}$ & 2.37 & 39.9 & 2.37 & 34.0 \\
\hline \multirow{4}{*}{$W e$} & Mode 1 & 0.10 & 1.73 & 0.10 & 1.47 \\
& Mode 2 & 5.30 & 88.4 & 5.30 & 75.3 \\
& Mode 3 & 204.6 & 3444 & 360.3 & 5156.7 \\
& & & & & \\
\hline
\end{tabular}

Table 3: We for contraction flow

\begin{tabular}{|c|c|c|c|}
\hline$Q m m^{3} / s$ & $\begin{array}{c}\Delta p^{E x p}(\mathrm{MPa}) \\
\text { LDPE }\end{array}$ & $\begin{array}{c}\Delta p^{\text {Sim }}(\mathrm{MPa}) \\
\text { Single mode }, \lambda_{1}^{\text {spec }}=5 s\end{array}$ & $\begin{array}{c}\Delta p^{\text {Sim }}(\mathrm{MPa}) \\
\text { Multi-mode }\end{array}$ \\
\hline 2.37 & 3.27 & 4.09, fluid $C_{1}$ & $5.90, G_{1}, \epsilon=1.0$ \\
& & $W e=30.18, \epsilon=0.15, \xi=0.02$ & $4.00, G_{2}, \epsilon=2.5$ \\
\hline 39.9 & 10.9 & 11.7, fluid $D_{2}$ & $10.3, G_{1}, \epsilon=1.0$ \\
& & $W e=508.0, \epsilon=0.3, \xi=0.02$ & \\
\hline
\end{tabular}

Table 4: Pressure drop for contraction flow, LDPE polymer 


\begin{tabular}{|c|c|c|}
\hline$Q m m^{3} / s$ & $\begin{array}{c}\Delta p^{E x p}(\mathrm{MPa}) \\
\text { HDPE }\end{array}$ & $\Delta p^{\text {Sim }}(\mathrm{MPa})$ \\
\hline 2.37 & 5.83 & $\begin{array}{l}7.40, \text { fluid } H_{2}, \epsilon=2.5 \\
\end{array}$ \\
\hline 34.0 & 19.90, fluid $H_{3}, \epsilon=3.0$ \\
\hline
\end{tabular}

Table 5: Pressure drop for contraction flow, HDPE polymer, multi-mode

\begin{tabular}{|ccccc|}
\hline ABB'A' & BCC'B' & CDD'C' & DEE'D' & EFF'E' \\
\hline 680 & 680 & 120 & 800 & 400 \\
\hline
\end{tabular}

Table 6: Number of elements per region

\begin{tabular}{|c|c|cc|cc|}
\hline & & LDPE & & HDPE & \\
\hline & & $1 Q$ & $2 Q$ & $1 Q$ & $2 Q$ \\
\hline \multirow{3}{*}{$W e$} & Mode 1 & 0.095 & 0.19 & 0.095 & 0.19 \\
& Mode 2 & 4.843 & 5.686 & 4.843 & 5.686 \\
& Mode 3 & 188.7 & 377.4 & 332.3 & 664.6 \\
\hline
\end{tabular}

Table 7: We for tube-tooling flow

\begin{tabular}{|l|c|cc|cc|}
\hline & & LDPE & & HDPE & \\
\hline & & $1 Q$ & $2 Q$ & $1 Q$ & $2 Q$ \\
\hline$\Delta p(\mathrm{MPa})$ & Single mode & 7.49 & 11.2, fluid $C_{1}$ & ---- & ---- \\
& Multi-mode & 6.90 & 8.40, fluid $G_{1}$ & 12.4 & 15.4 , fluid $H_{1}$ \\
\hline
\end{tabular}

Table 8: Pressure drop for tube-tooling flow 


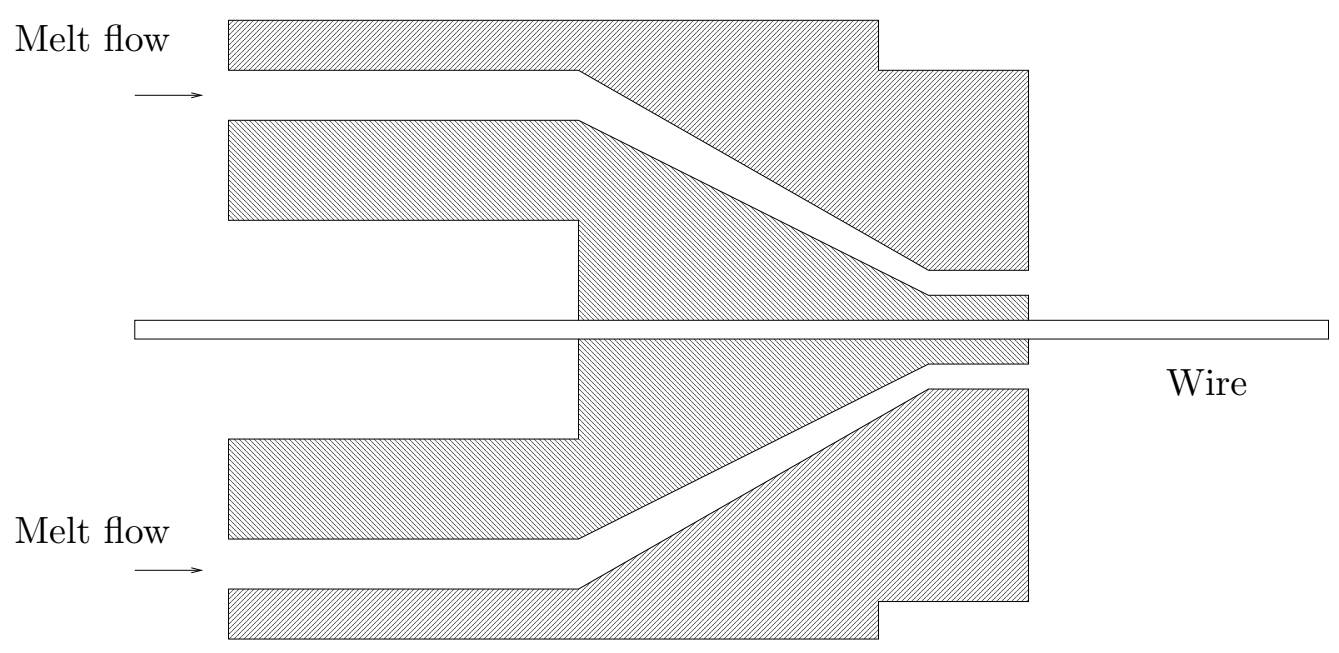

a) Tube-tooling die

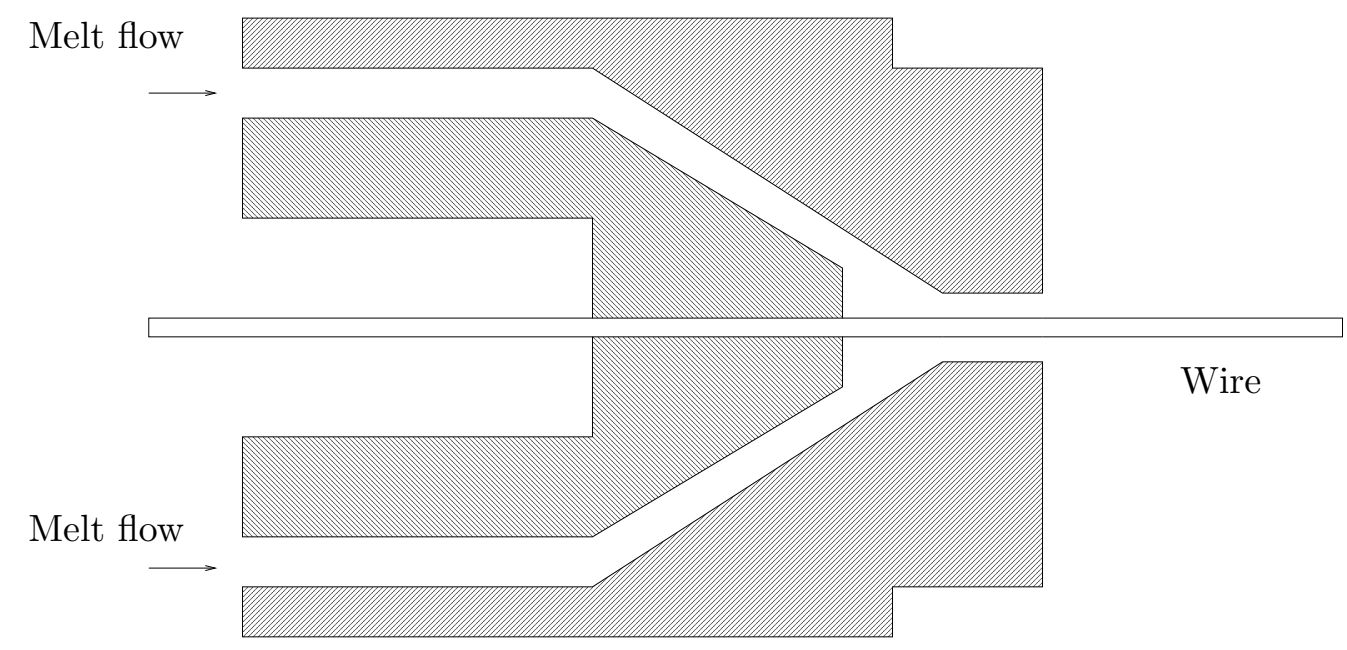

b) Pressure-tooling die

Figure 1: Schematic diagram of wire coating dies: (a) tube-tooling die; (b) pressure-tooling die. 


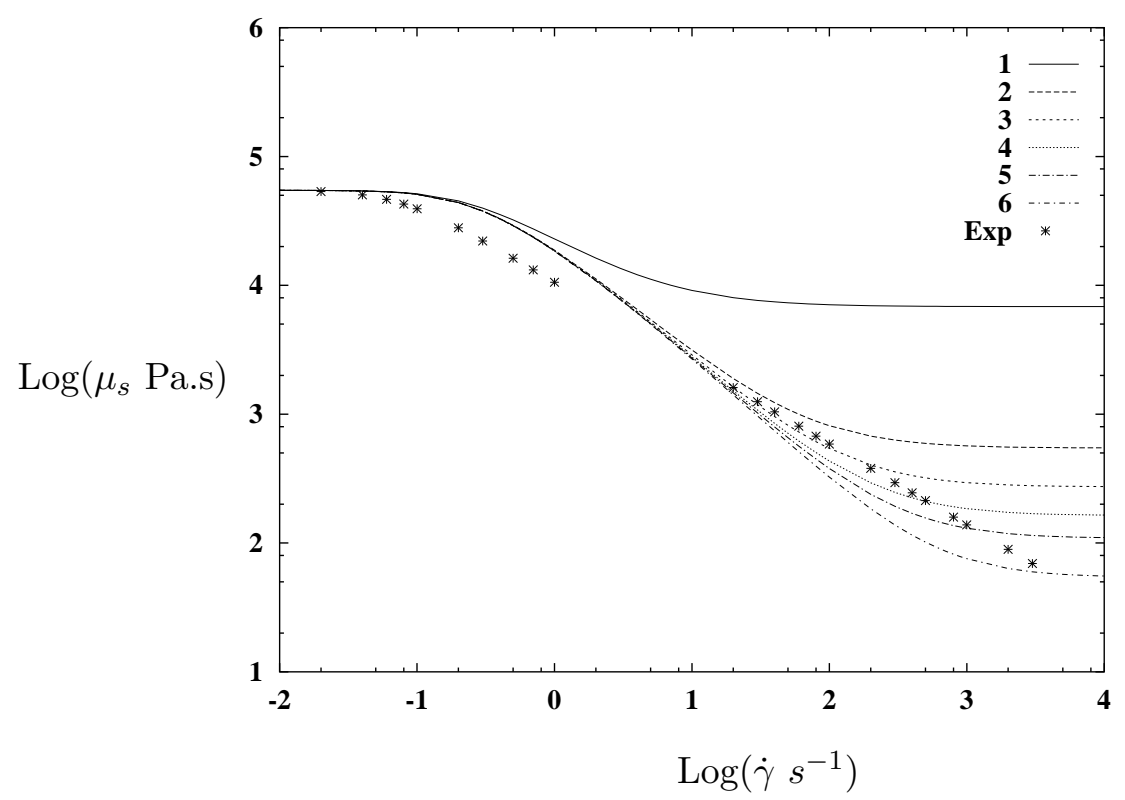

[1] $\mu_{1}=0.875$

[2] $\mu_{1}=0.99$

[3] $\mu_{1}=0.995$

[4] $\mu_{1}=0.997$

[5] $\mu_{1}=0.998$

[6] $\mu_{1}=0.999$

a) Shear viscosity

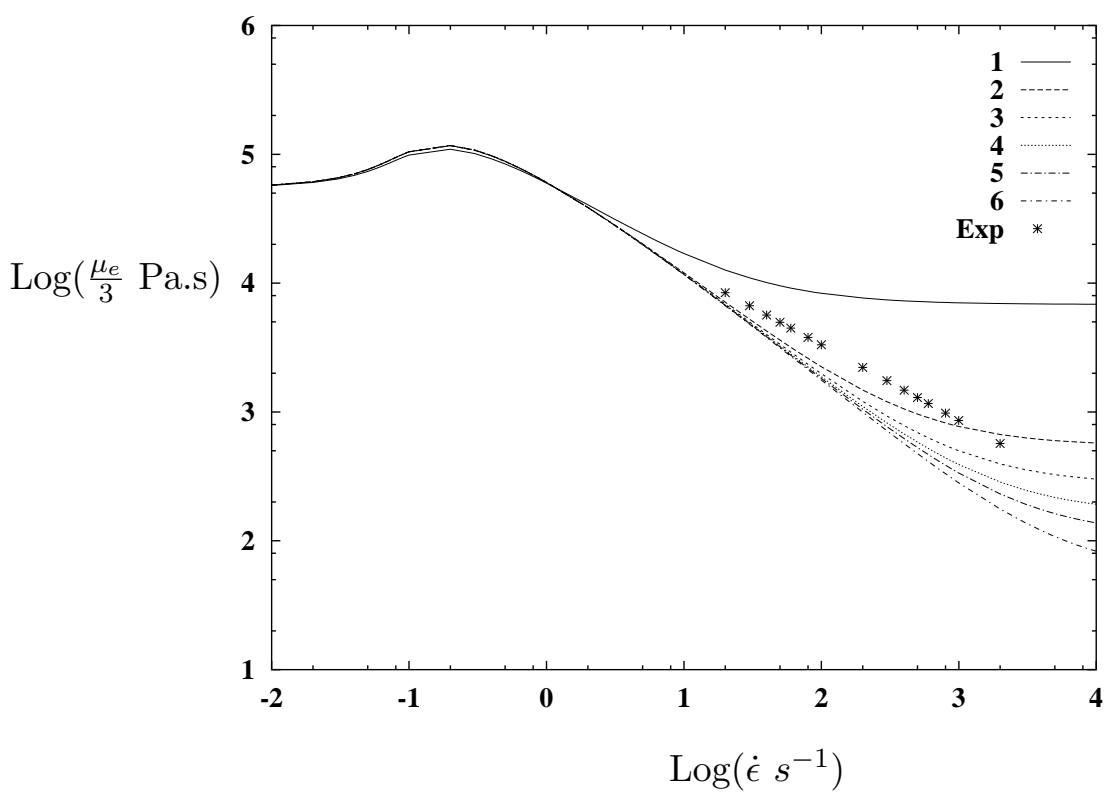

b) Elongational viscosity

Figure 2: Shear and elongational viscosities fits; $\mu_{1}$ variation, $\epsilon=0.15, \xi=0.02$. 


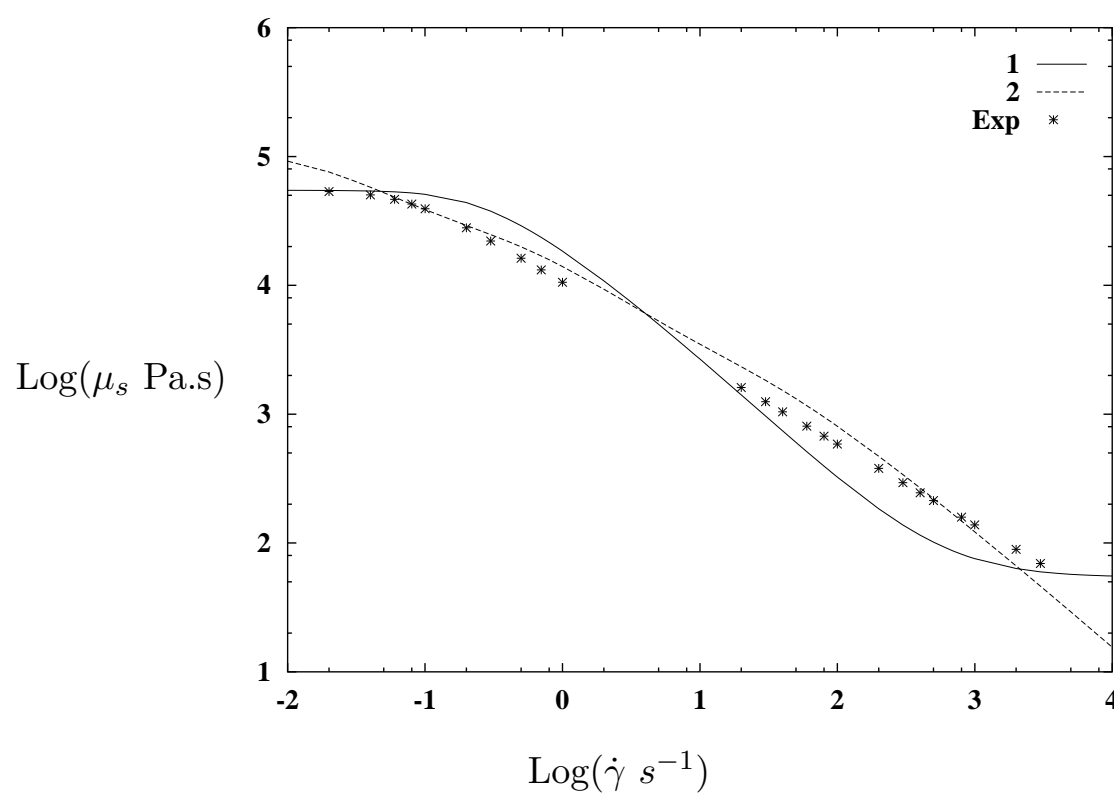

[1] 1-mode $\mu_{1}=0.999$, $\epsilon=0.15, \xi=0.02$

[2] 3-mode $\epsilon=1.0$, $\xi=0$.

a) Shear viscosity

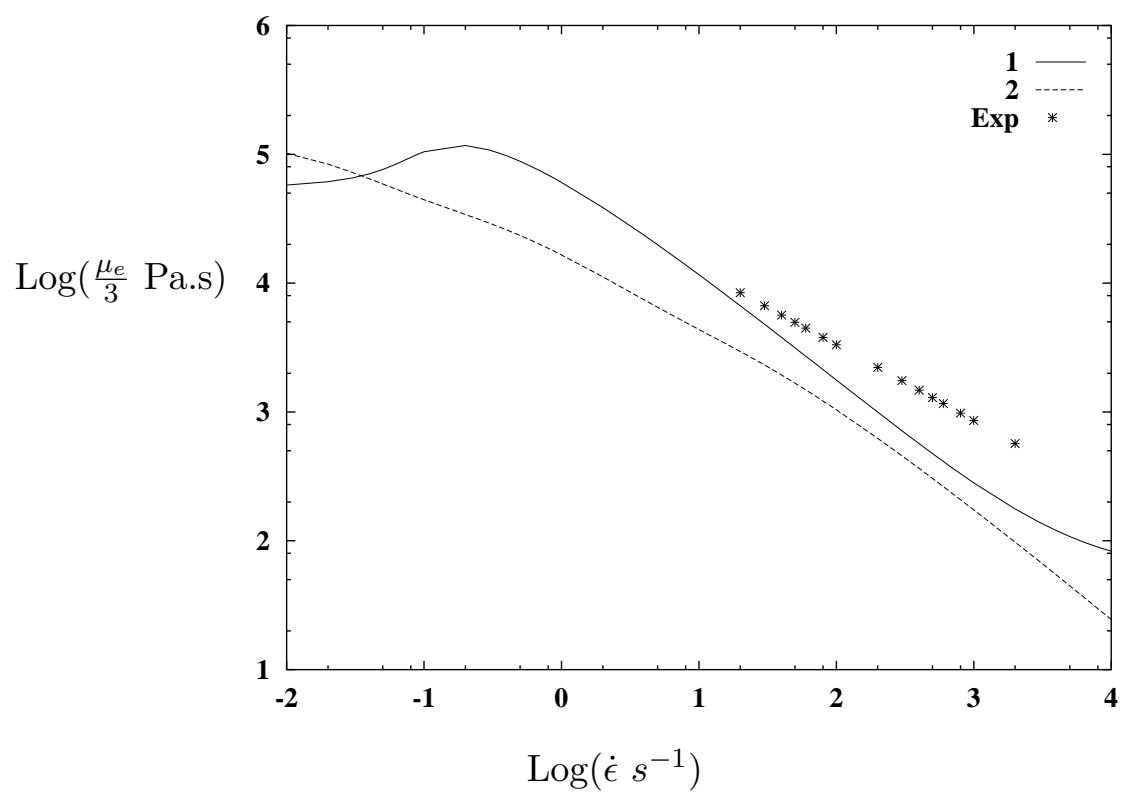

b) Elongational viscosity

Figure 3: Single and multi-mode fits for LDPE. 


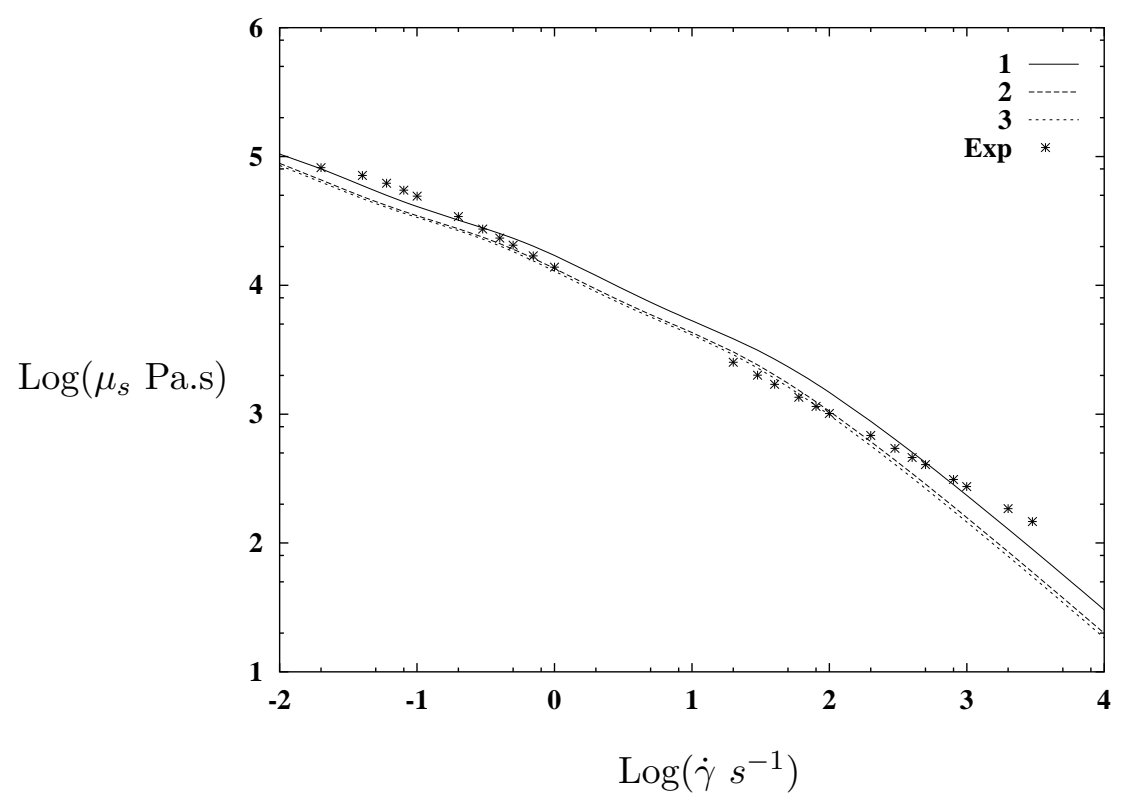

[1] $\epsilon=1.0$

$[2] \epsilon=2.5$

$[3] \epsilon=3.0$

a) Shear viscosity

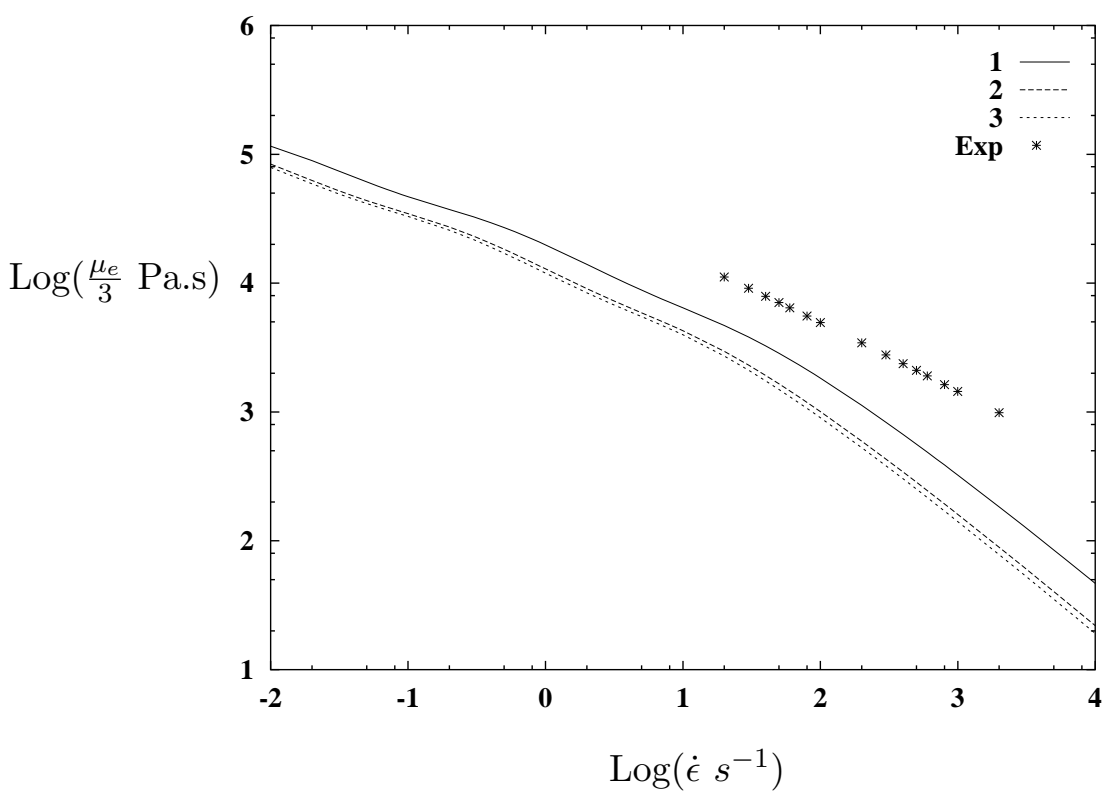

b) Elongational viscosity

Figure 4: Rheometrical data fits with $\epsilon$ variation for 3-mode HDPE. 


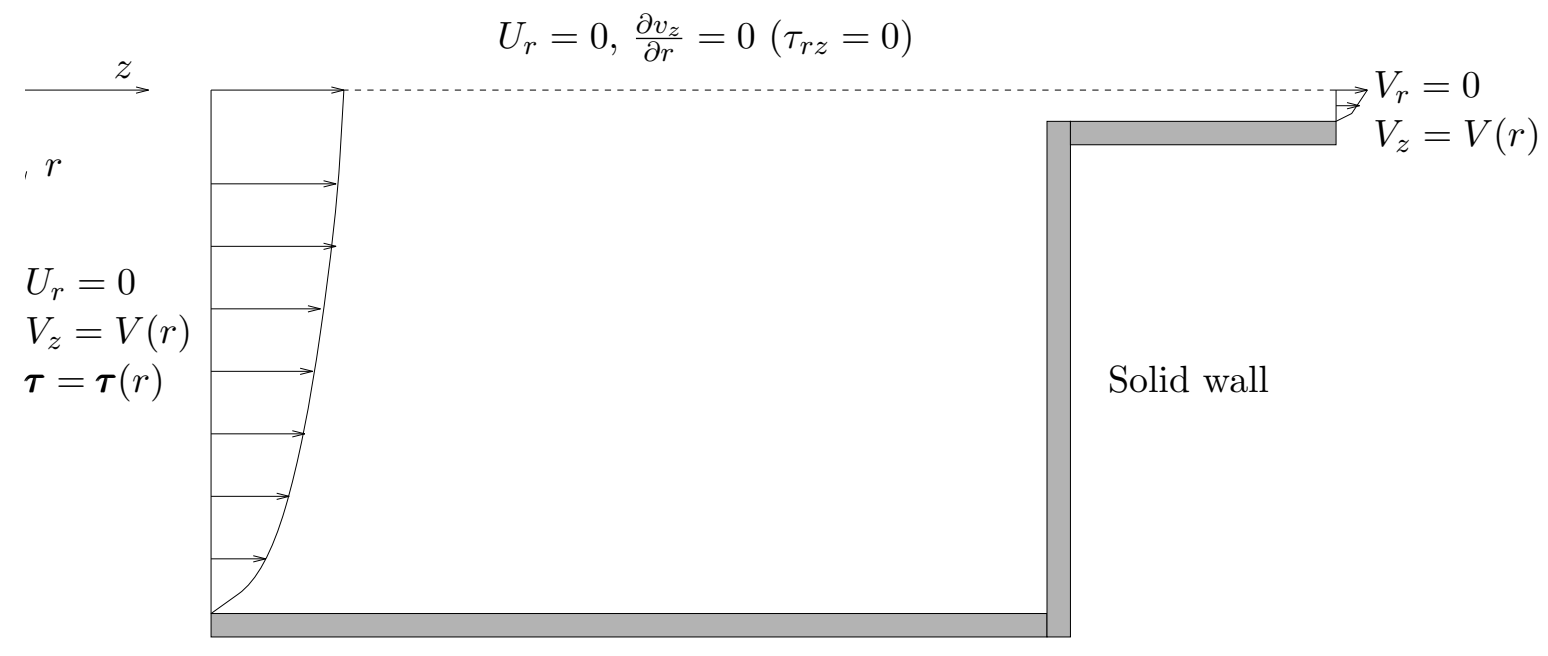

a) Schematic flow diagram

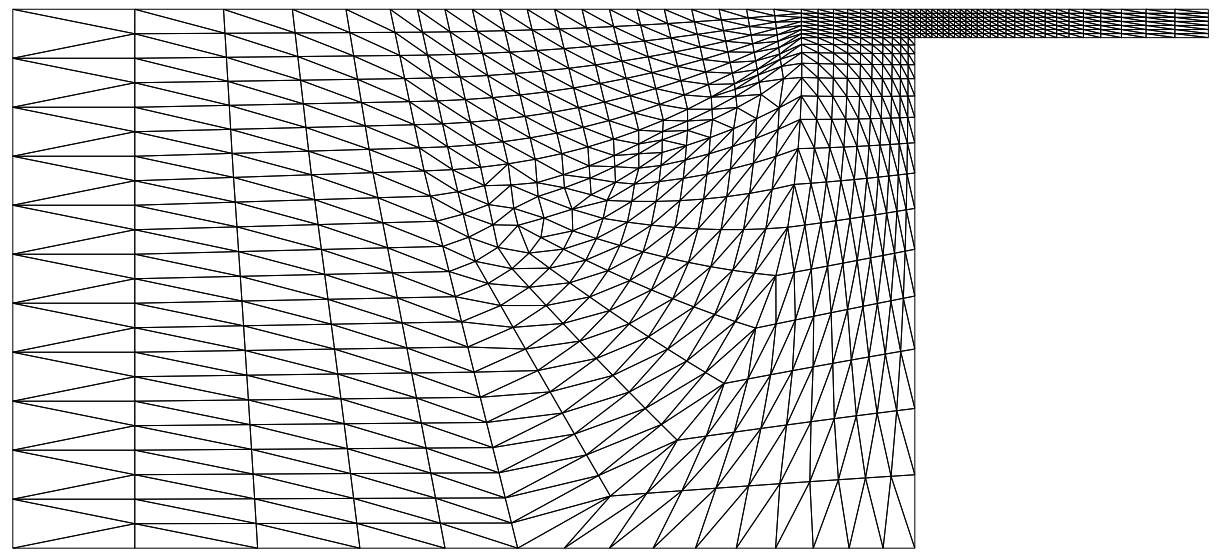

b) Finite element mesh

Figure 5: Schematic flow diagram and finite element mesh for 19:1 contraction geometry. 


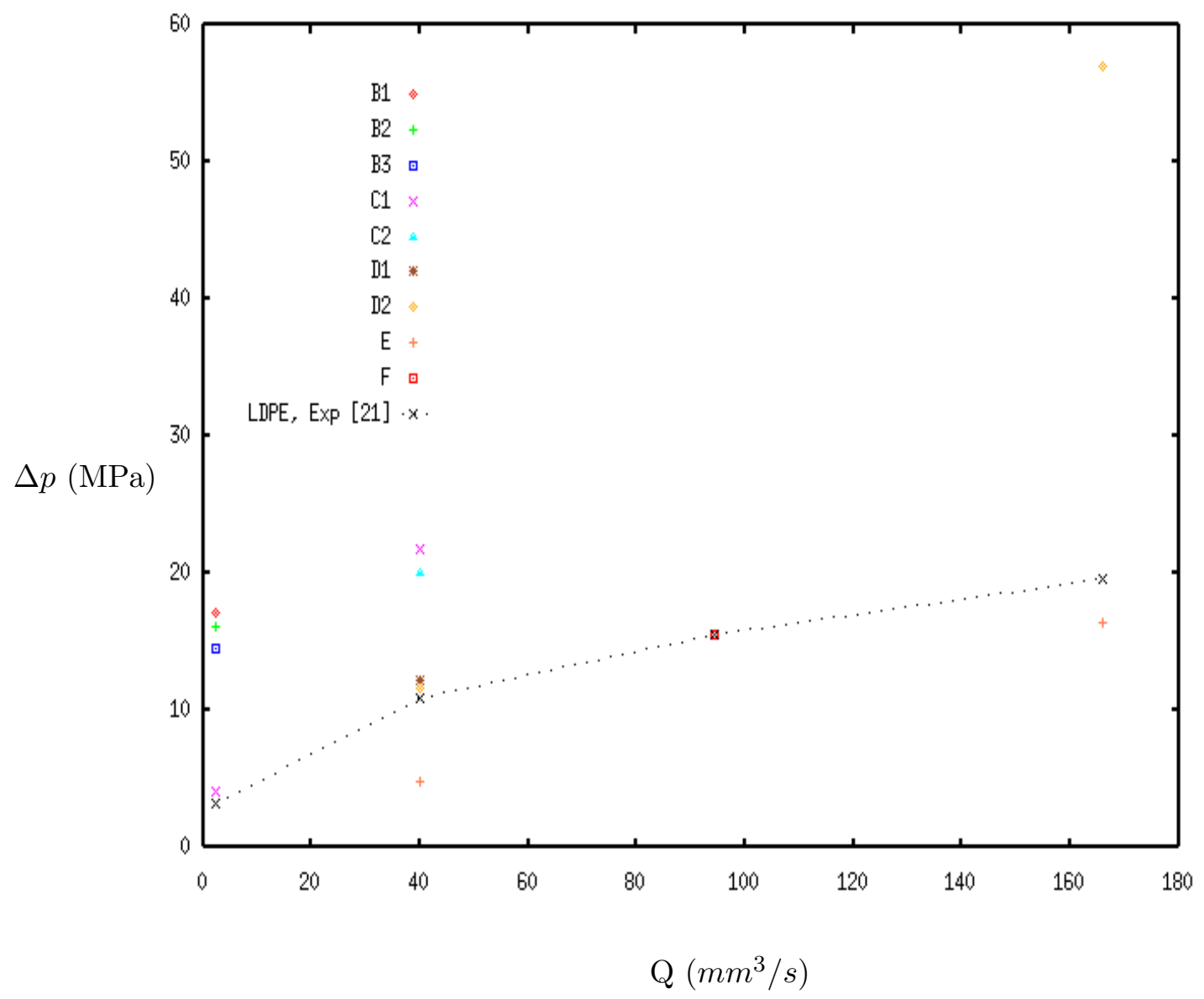

Figure 6: Pressure drop v flowrate for contraction problem, single mode model 


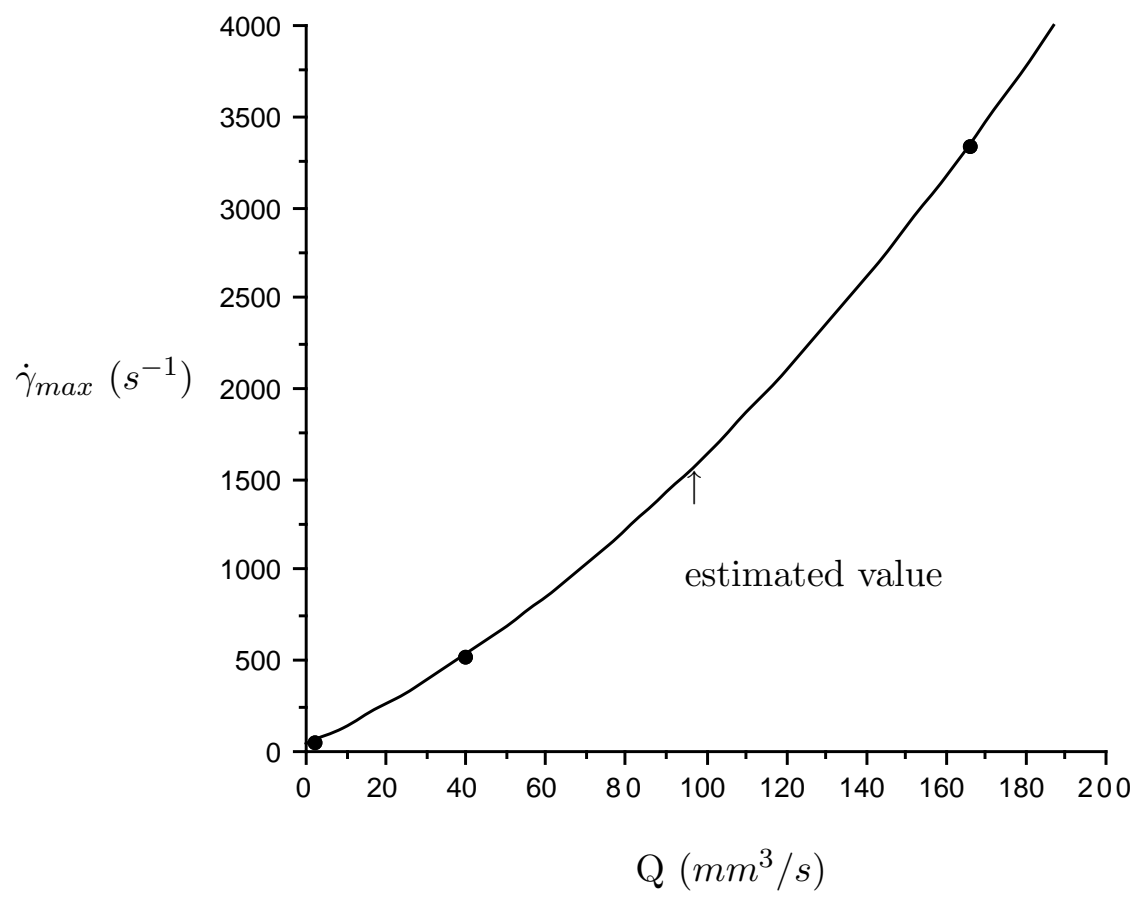

Figure 7: Maximum shear-rate v flowrate correlation for contraction problem, LDPE 


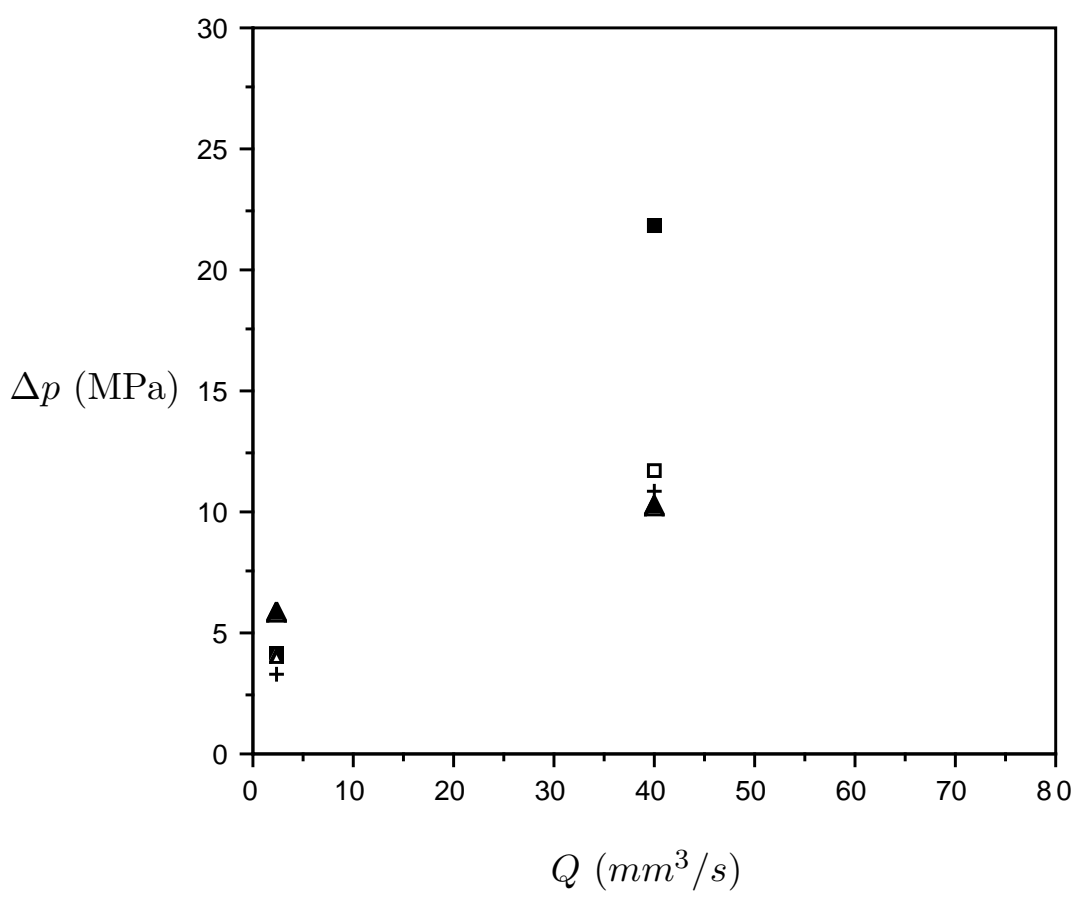

- $\mu_{1}=0.99, \epsilon=0.15, \xi=0.02$

ㅁ $\mu_{1}=0.995, \epsilon=0.3, \xi=0.02$

$\Delta 3$-modes, $\epsilon=1.0$

$\Delta$ 3-modes, $\epsilon=2.5$

$+\operatorname{Exp}[21]$

a) LDPE

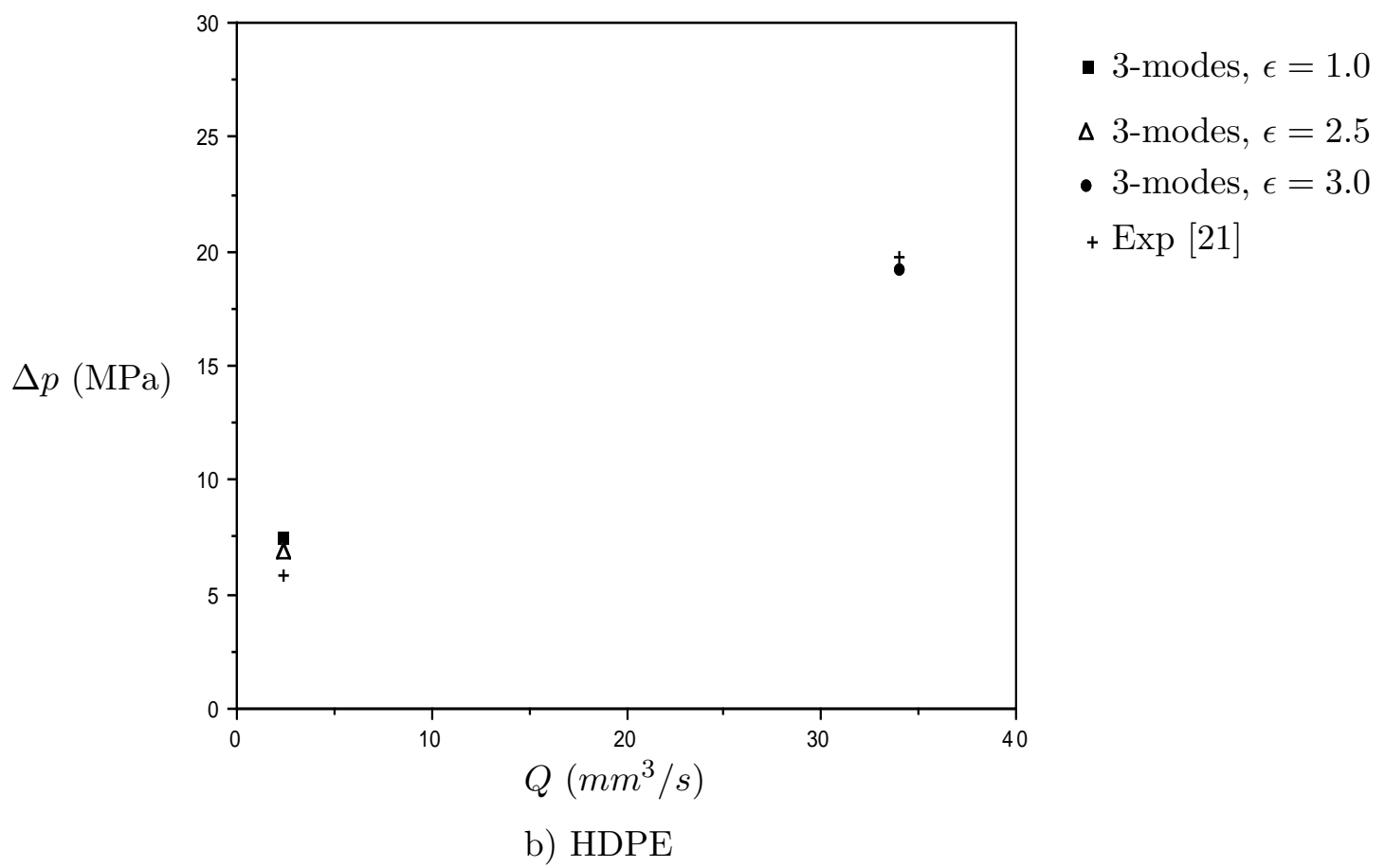

Figure 8: Simulated and experimental pressure drops for contraction flow; a) LDPE, b) HDPE 


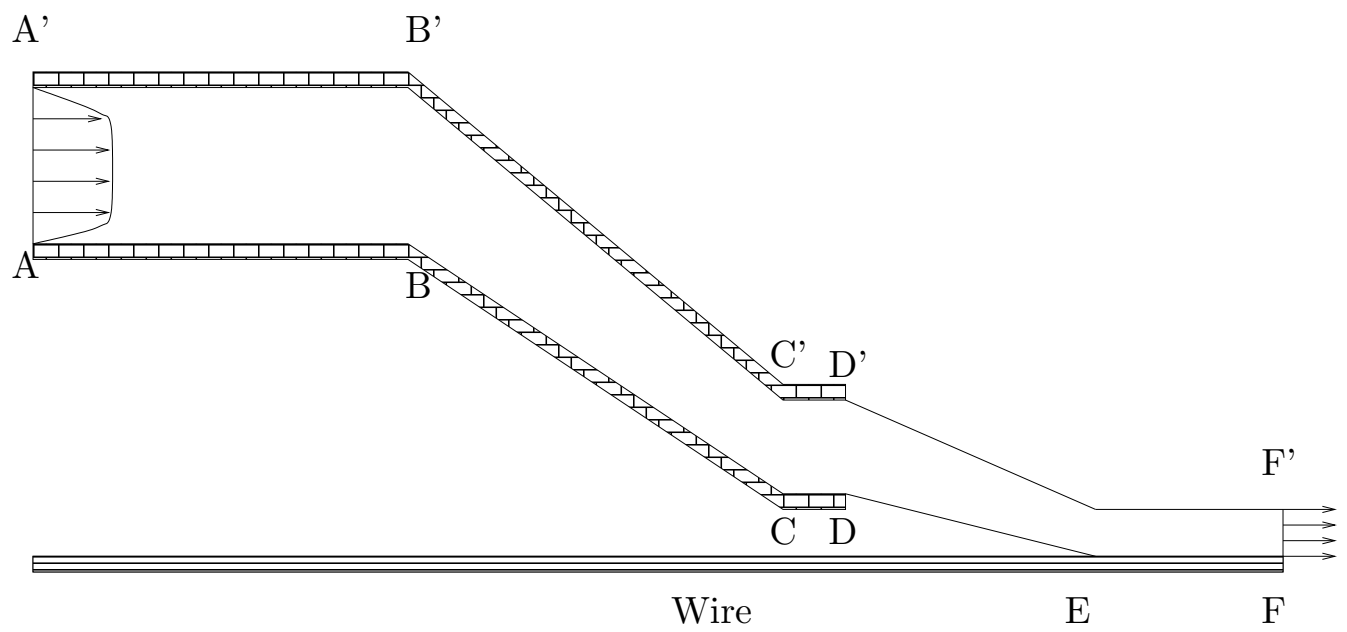

a) Schematic flow diagram

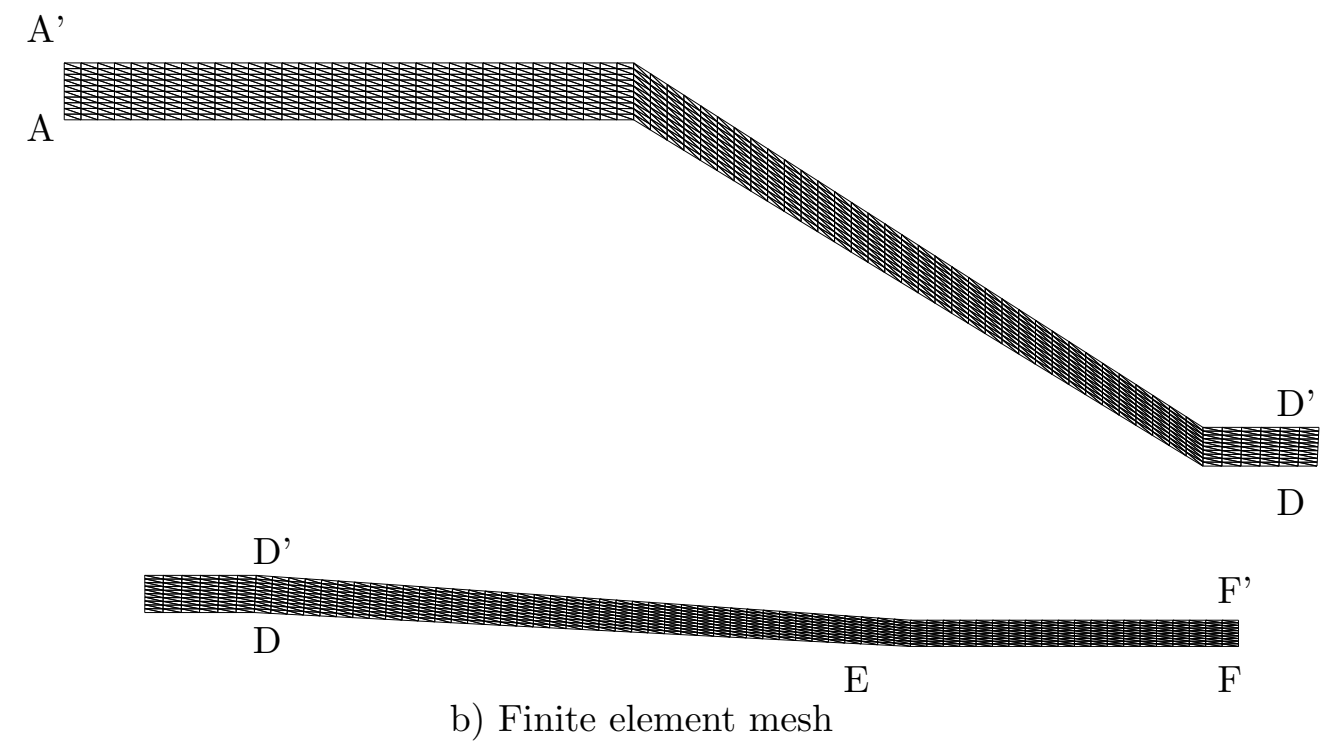

Figure 9: Schematic diagram and finite element mesh for wire coating tube-tooling die. 


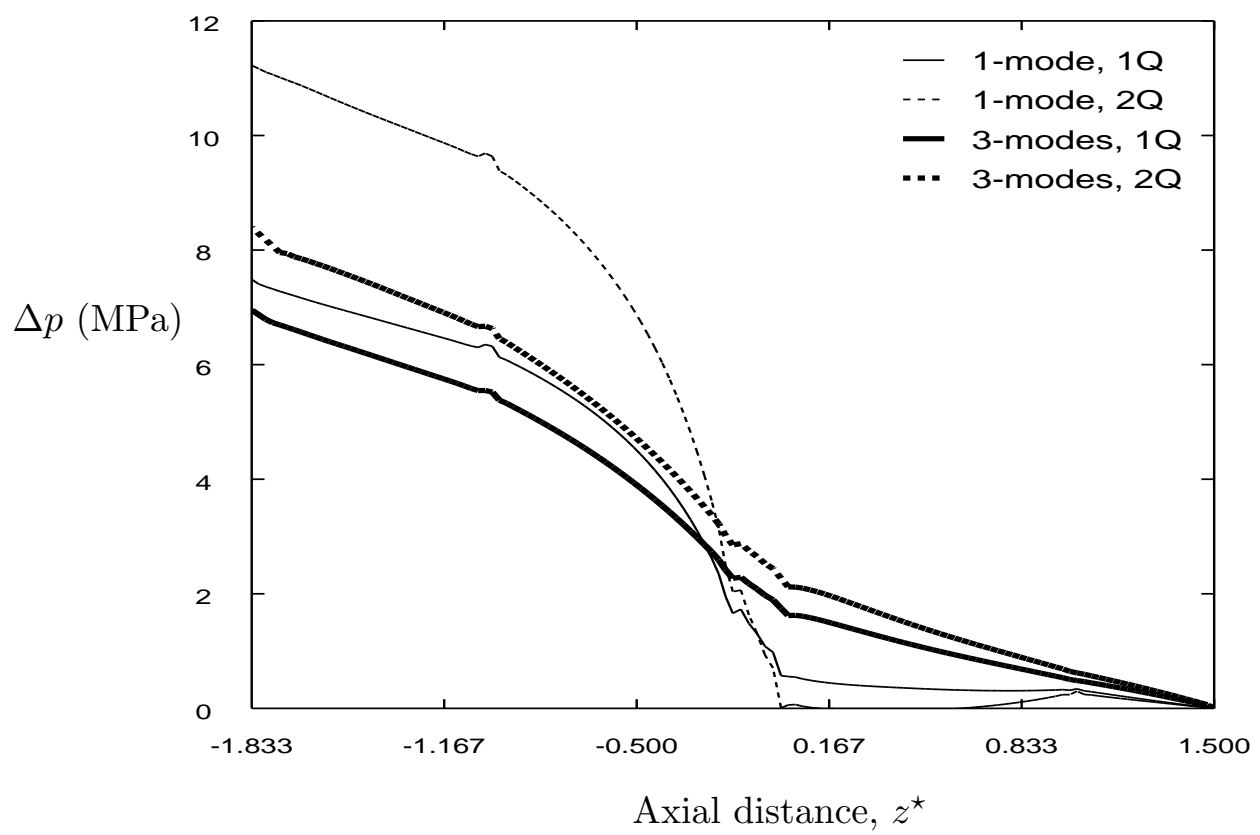

Figure 10: Tube-tooling flow, streamwise pressure profiles, single and multi-mode models, LDPE 

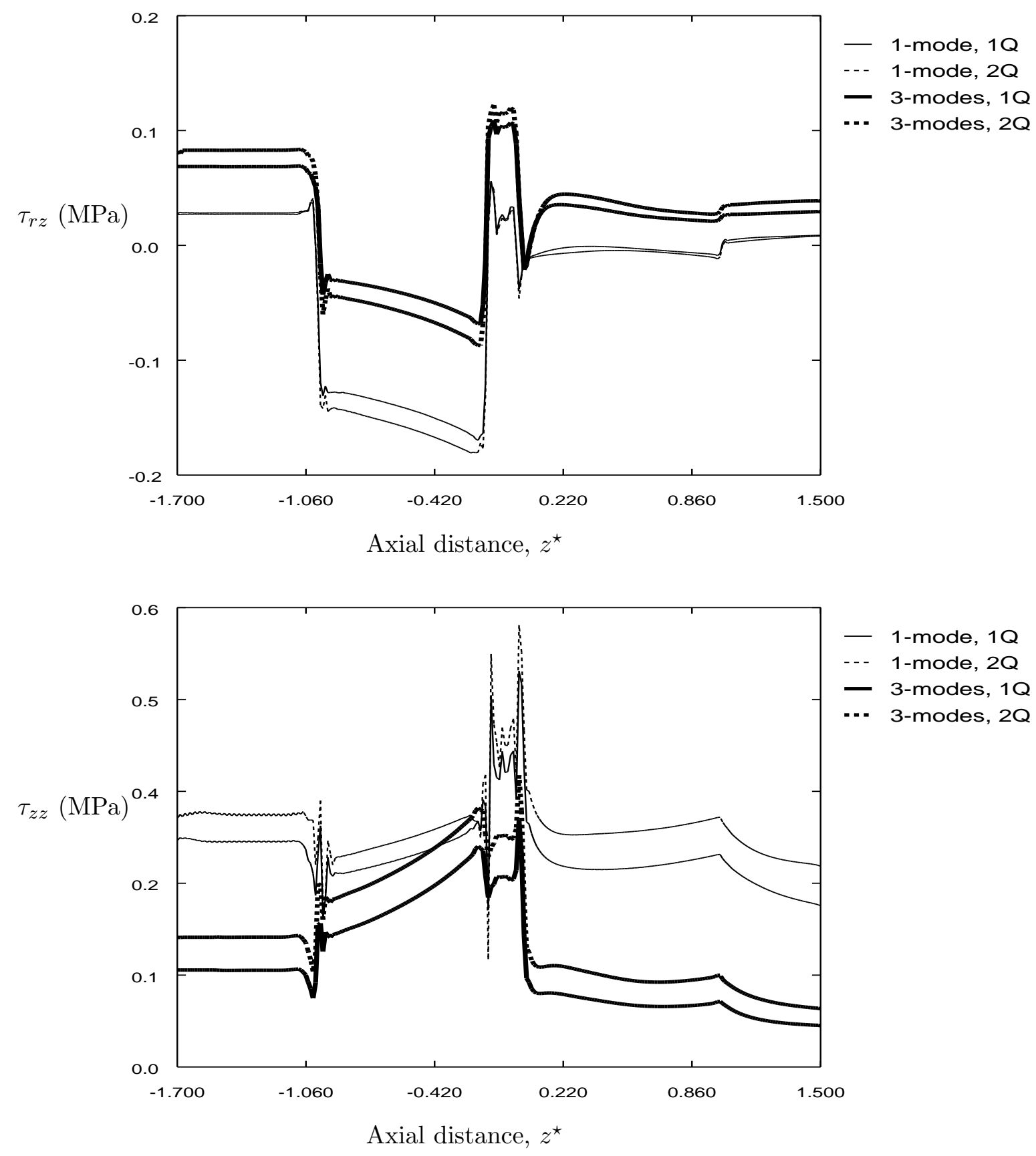

Figure 11: Tube-tooling flow, streamwise stress profiles, single and multi-mode models, LDPE, a) $\tau_{r z}$, b) $\tau_{z z}$ 


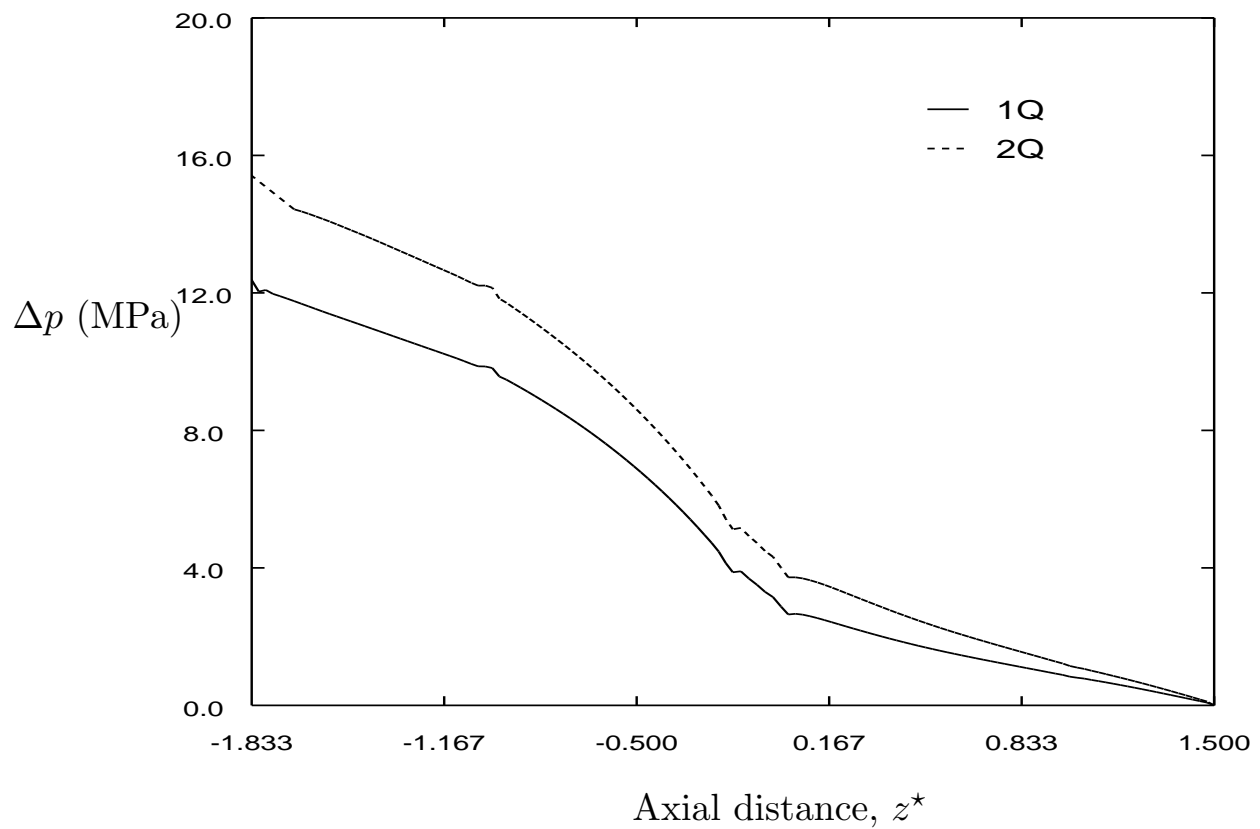

Figure 12: Tube-tooling flow, streamwise pressure profiles, standard and double flowrate, HDPE 

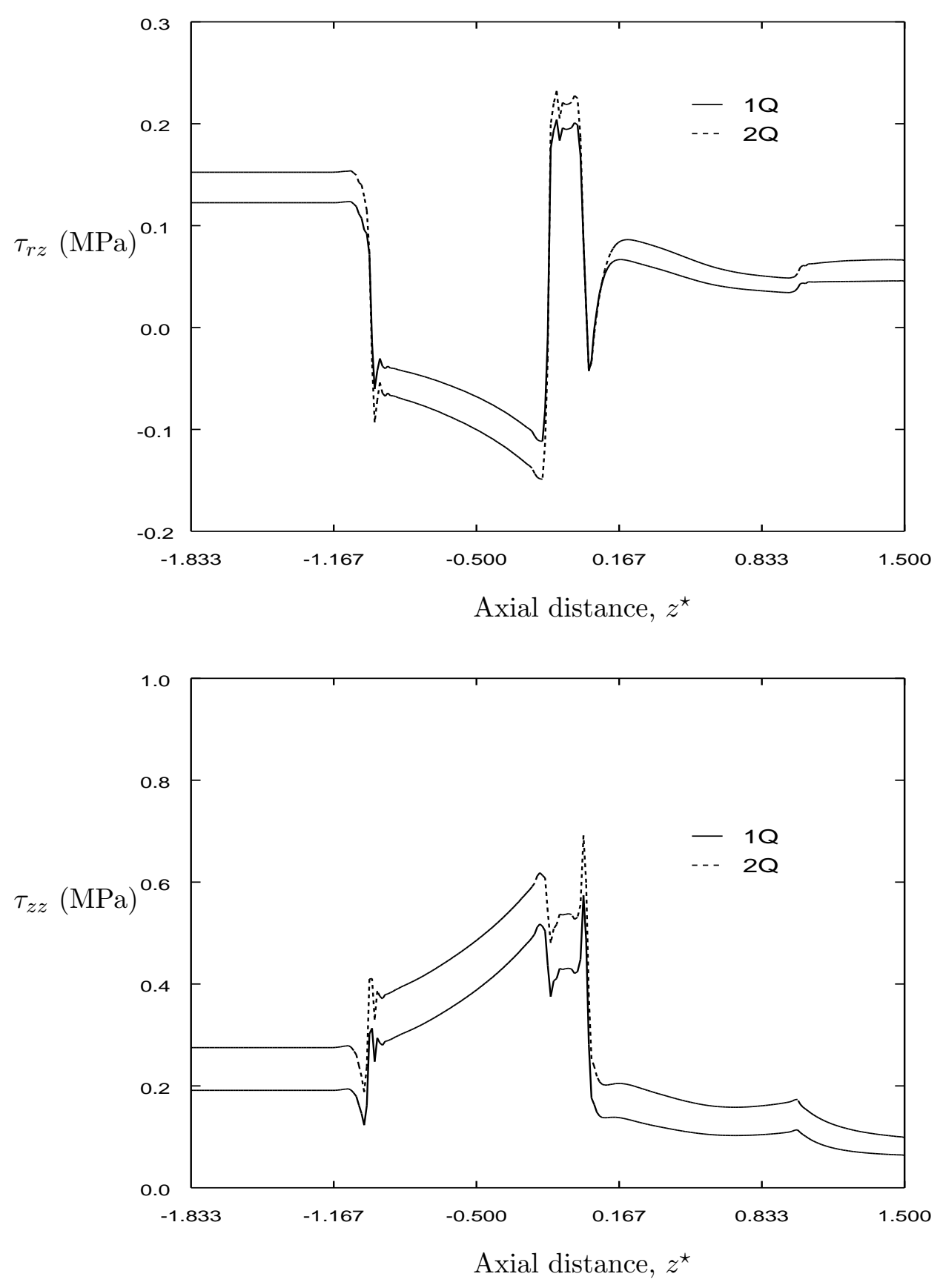

Figure 13: Tube-tooling flow, streamwise stress profiles, multi-mode, HDPE, a) $\tau_{r z}$, b) $\tau_{z z}$ 

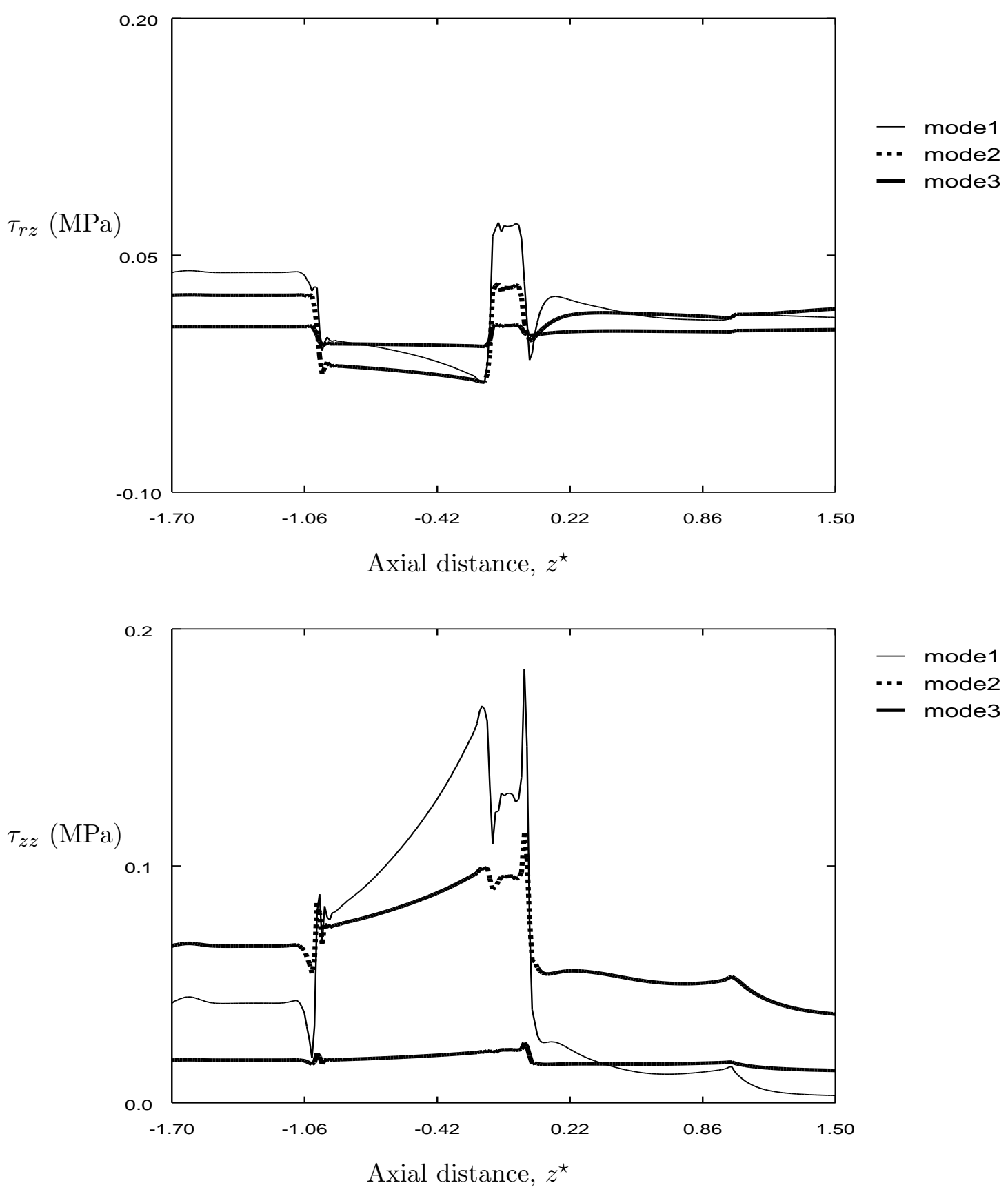

Figure 14: Tube-tooling flow, streamwise stress profiles for individual stress mode, LDPE, a) $\tau_{r z}$, b) $\tau_{z z}$ 

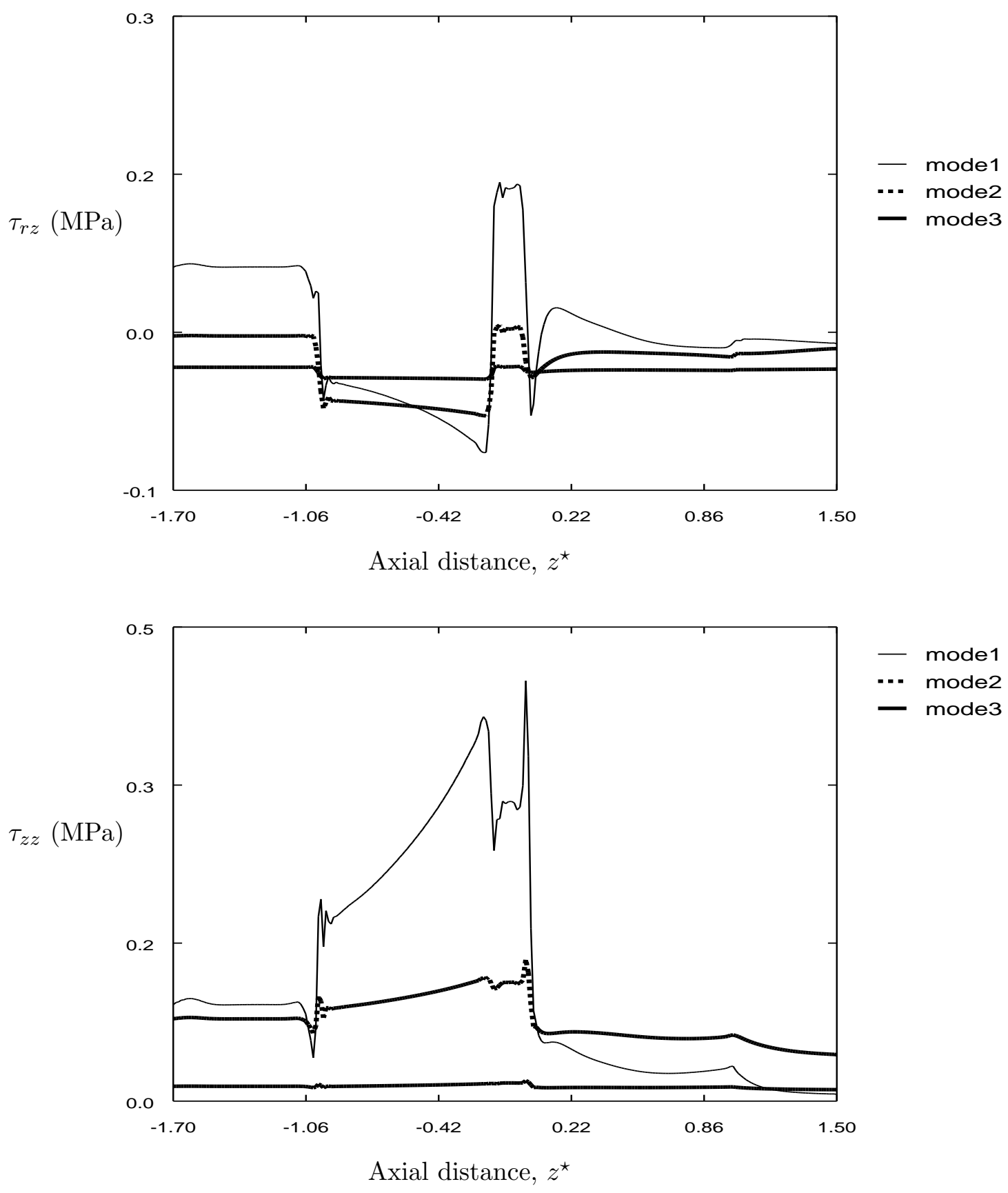

Figure 15: Tube-tooling flow, streamwise stress profiles for individual stress mode, HDPE, a) $\tau_{r z}$, b) $\tau_{z z}$ 